\title{
Correspondance de Charles Darwin et d'Alphonse de Candolle
}

\author{
Par Charles Baehni, Genève
}

\section{Introduction}

On peut dire, sans doute, que Darwin et Alphonse de Candolle se sont beaucoup occupés l'un de l'autre; rien de plus naturel, d'ailleurs, puisque tous deux ont eu, à un très haut degré, le goût des idées générales, et que tous deux ont possédé la patience géniale de s'astreindre à des travaux minutieux, longs dépouillements et interminables expériences, destinés à étayer leurs théories. Tous deux encore ont eu le sens épistolaire bien développé, ce qui fait que nous possédons, de chacun, une volumineuse correspondance et, heureusement pour nous, une petite suite de lettres qu'ils ont échangées et qui présentent assez peu de lacunes, pour qu'elles méritent d'être publiées ensemble. Comme on va le voir, elles n'ont pas toutes la même densité et surtout, l'intérêt, comme il arrive toujours dans une correspondance qui n'était pas, à l'origine, destinée à la publication, ne se soutient pas tout au long. On aurait pu les tronquer et n'en publier que les passages qui pouvaient éclairer d'un jour nouveau les rapports des deux savants. On y a renoncé parce que, en procédant ainsi, le mouvement général de cette correspondance se serait perdu.

Les lettres de Darwin font partie des archives de la famille de Candolle; pour celles d'Alphonse de Candolle, on n'a que des copies d'une main inconnue, mais qui ont été revues et corrigées par leur auteur vers 1885. Apparemment, il avait redemandé ses lettres pour les faire copier au moment où Francis Darwin faisait de même pour celles de son père, en vue de leur publication (voir ci-dessous, note ${ }^{\mathrm{a}}$ ).

C'est le lieu de remercier chaleureusement Madame Augustin de CanDOLLE qui nous a aimablement communiqué une partie des richesses scientifiques qu'elle possède, ses deux filles, Mesdames Jean-Pierre Weber et Edmond Bovey qui ont déchiffré avec talent les lettres de Darwin (l'écriture est souvent presque illisible!), enfin son fils, Monsieur Roger DE Candolle qui nous a permis, grâce à sa connaissance parfaite de l'anglais, d'éclaircir plusieurs points obscurs.

La correspondance s'ouvre sur une lettre de Darwin datée du 11 novembre 1859 et elle se clôt vers l'époque de la mort de Darwin sur un billet de Candolle du 9 juillet 1881, c'est-à-dire qu'elle embrasse un peu plus de vingt années. Nous ne possédons plus la série complète: des lettres se sont cer- 
tainement perdues; d'autres sont peut-être restées sans réponse. Enfin, les deux hommes se sont vus, à deux reprises, en 1839 d'abord, puis en 1880, et nous n'avons pas un récit détaillé de leurs entretiens.

1859! Pour Darwin, c'est l'année cruciale, non pas celle qui devait déterminer sa carrière (cet événement prit place en 1831, lorsqu'il s'embarqua sur le Beagle pour son voyage autour du monde), mais celle où parut son ouvrage capital, On the origin of species by means of natural selection. On peut difficilement se rendre compte aujourd'hui de la confusion qui régnait alors dans l'esprit des botanistes, état qui n'est pas sans rappeler, d'ailleurs, et par bien des côtés, celui des botanistes actuels. On était loin de la période heureuse où le dogme - véritable article de foi - de la constance des espèces fournissait la base philosophique nécessaire aux recherches sur les plantes. Cette période avait pris fin avec Linné, disons avec le Linné du Systema, du Genera et du Species tout imprégnés des doctrines scholastiques et qui servirent de base aux travaux ultérieurs, mais non pas du Linné des Fragmenta (publiés tout d'abord dans Classes Plantarum qui ont paru à Leyde en 1738) qui ouvrait la voie aux Jussieu. L'idée de parenté, d'une parenté réelle mais fort reculée dans le temps, ne s'était pas encore présentée aux botanistes et il n'était donc pas encore question de penser, tout au moins d'une façon claire, à une évolution des êtres organisés. La seule parenté qu'on entrevoyait était celle que pouvaient avoir entre elles les «idées» dans le sens platonicien et qu'on situait hors du monde matériel. Les botanistes se trouvaient donc astreints à décrire toutes les formes nouvelles qui leur tombaient sous la main, à dresser en quelque sorte le catalogue descriptif des formes vivantes, et à insérer les produits de leur industrie dans les casiers de la systématique artificielle; la botanique se trouvait par conséquent ravalée à un niveau si bas qu'on hésite aujourd'hui à lui donner le nom de science.

Viennent Robert Brown, les Jussieu, Lamarck, A.-P. de Candolle: sans cesser de décrire des groupes nouveaux, dégageant notamment la notion de famille, ils fondent le système qu'on appelle (improprement) naturel. On admet encore la notion de fixité des espèces, mais on tient compte, largement dans certains cas, des idées d'avortement, d'adhérence, de dégénérescence, en somme, on reconnaît l'existence de phénomènes qui impliquent un développement: c'était introduire le loup dans la bergerie, c'était préparer les bases de la théorie de la descendance, négation même du dogme de la fixité. Mais, pendant une longue période, la tension des esprits, tiraillés entre deux tendances, ne fit que croître: d'un côté les botanistes s'ac- 
crochaient désespérément à l'idée de la fixité, d'un autre, ils étaient entraînés par le courant vers l'idée du mouvement. Les deux états sont notoirement inconciliables, mais le choix était cruel, car si l'on possédait une théorie rendant compte de la fixité, on n'en avait point pour expliquer l'évolution: aussi, vers le milieu du siècle, les biologistes étaient-ils bien préparés à recevoir l'Origine des espèces.

Ce livre, arrivant comme il le fit, dans un monde scientifique momentanément privé d'idées directrices, eut un très grand retentissement. Non seulement Darwin y apportait la preuve que la constance des espèces n'était qu'un article de foi, non vérifiable par conséquent, mais il montrait que la parenté en quelque sorte idéale entrevue par les systématiciens de l'école de Jussieu et si fermement démontrée par A.-P. de Candolle était ou plutôt devait être une réalité (si l'on ose dire, en simplifiant beaucoup les choses et en accouplant des mots qui vont mal ensemble) en quelque sorte matérielle. Les degrés de parenté décelés par les systématiciens devenaient des états - provisoires peut-être - d'une évolution continue. Darwin, d'un seul coup, justifiait les travaux des systématiciens en leur donnant les bases théoriques qui leur avaient fait défaut pendant si longtemps, et donnait un sens aux recherches qui suivirent.

Quelle était, à ce moment, la position d'Alphonse de Candolle dans ce tumulte d'idées? En 1859, il avait déjà derrière lui une belle carrière de botaniste; aux yeux de Darwin, il devait presque faire figure de patriarche, un patriarche de 53 ans, et qui avait publié près de cent travaux dont le plus important était certes, à l'époque, sa Géographie botanique raisonnée. Elevé à l'école de son père (lui-même disciple authentique de Jussieu), il ne semble pas avoir été troublé par les difficultés, mais il les voyait d'un œil clair. En 1855, donc quatre ans avant l'Origine des espèces, il admettait dans sa Géographie botanique (ch. 11, p. 1056 et suivantes) une création, dans certains cas, de nouvelles formes spécifiques dérivées des anciennes. [II prouvait,] en outre, que la majorité des espèces remonte à des temps plus reculés qu'on ne supposait et qu'elles ont traversé des changements géologiques ou climatériques ${ }^{\text {a }}$. Mais presque au même moment, exactement deux ans plus tard ${ }^{\mathrm{b}}$, prenant part au débat passionné qui suivit la présentation par Payer de son ouvrage sur l'Organisation comparée de la Fleur et où Flourens, le Prince Bonaparte, Quatrefages et Brongniart s'affrontèrent autour de

a Alphonse de Candolle, Darwin considéré au point de vue des causes de son succès, p. 14, $2^{\mathrm{e}}$ éd., Genève 1882.

b C.R. Acad. Sci. (Paris), séance du 30 mars 1857. 
l'héritage contesté de Laurent de Jussieu, Alphonse de Candolle - comme tous les autres d'ailleurs - n'évoque même pas l'idée d'un changement possible, l'idée de variation ou celle d'évolution et semble ignorer par conséquent, le rôle du temps que Darwin devait faire intervenir avec tant d'éclat.

Il n'est pas sans intérêt, avant d'ouvrir ces lettres, de noter qu'en les publiant, on ne fait qu'obéir à un vœu d'Alphonse de Candolle ${ }^{\mathrm{c}}:$ " $J^{\text {' } a i ~ l i e u}$ d'espérer qu'on publiera tout ou partie des lettres de Darwin. Elles feraient bien ressortir l'excellence de son caractère, toujours noble, modeste et aimable. On verrait à quel point il s'intéressait aux travaux des autres. Chez lui, le plaisir de rendre justice à chacun était poussé à un rare degré. Jamais aussi il n'avait la moindre trace de ces petites jalousies ou susceptibilités qui déplaisent quelquefois chez les hommes supérieurs.»

La correspondance de Darwin a été publiée en partie ${ }^{\mathrm{d}}$ et plusieurs des lettres qu'on trouvera plus loin y figurent déjà. Il restait à les mettre en regard de celles qu'il avait reçues d'Alphonse de Candolle.

Note: Sauf indication contraire, les lettres de Darwin sont datées de Down, Bromley, Kent; le millésime a été ajouté par Alphonse de Candolle et les lettres de ce dernier sont datées de Genève.

\section{Darwin à Candolle}

Nov. 11th [1859]1

Dear Sir,

I have thought that you would permit me to send you (by Messrs Williams \& Norgate Booksellers) a copy of my work (as yet only an abstract) on the Origin of Species ${ }^{2}$. I wish to do this, as the only though quite inadequate manner, by which I can testify to you, the extreme interest which I have felt, and the great advantage, which I have derived, from studying your grand and noble work on Geographical Distribution ${ }^{3}$. Should you be induced to read my volume, I venture to remark that it will be intelligible only by reading the whole straight

c Alphonse de Candolle, Darwin, etc., p. 40.

d The life and letters of Charles Darwin, edited by his son Francis Darwin, 3 vol., London 1888. - More letters of Charles Darwin, edited by Francis Darwin, 2 vol., London 1903.

${ }^{1}$ Lettre publiée dans Life and letters 2, 216.

${ }^{2}$ On the origin of species by means of natural selection, Murray, London 1859.

${ }^{3}$ Géographie botanique raisonnée, Masson, Paris, et Keßmann, Genève 1855. 
through, as it is very much condensed. It would be a high gratification to me, if any portion interested you. But I am perfectly well aware, that you will entirely disagree with the conclusion, at which I have arrived.

You will probably have quite forgotten me; but many years ago you did me the honour of dining at my house in London to meet M. \& Mad. Sismondi,the uncle and aunt of my wife. With sincere respect, I beg leave to remain,

$$
\text { Yours very faithfully, }
$$

Charles Darwin

A maintes reprises, dans cet ouvrage fondamental, Darwin, en effet, s'est inspiré des travaux de son correspondant. Il le cite à propos de la distribution des plantes de petite taille, faisant allusion à la loi formulée par Candolle (Geogr. bot. p. 532): l'aire moyenne des espèces du règne végétal est d'autant plus grande que leur taille moyenne est plus petite; il le cite encore au sujet de la variabilité des plantes à grande aire d'extension, sur le fait (observé par A.DC.) que les graines ailées ne se trouvent jamais dans les fruits qui ne s'ouvrent pas, ce qui s'explique aisément, dit Darwin, puisque l'acquisition d'ailes ne pourrait se faire graduellement pour des graines qui restent enfermées; il le cite encore au sujet de la disparition brusque, à certaines altitudes, d'espèces alpines communes, sur la végétation des îles et celle de l'Australie, sur la distribution des espèces aquatiques. On observe que chez Darwin il n'y a ni critique, ni commentaires superflus; simplement il glane dans la géographie botanique tous les faits dont il a besoin et les emploie pour étayer sa théorie. La carrière d'Alphonse de Candolle est la preuve que la crainte exprimée par Darwin (but I am perfectly well aware ...) était sans fondement; Candolle l'a chicané, comme on sait sur les détails, mais il a accepté l'ensemble de la théorie, comme on le verra peu à peu dans cette correspondance et dans ses ouvrages ultérieurs.

En lisant attentivement l'ensemble des lettres qui vont suivre, on se rendra parfaitement compte que les influences respectives des deux hommes l'un sur l'autre ne se sont pas exercées au même moment. En écrivant son Origin of species, Darwin avait devant lui la Géographie botanique dont l'auteur jouissait depuis longtemps d'une autorité scientifique considérable. On vient de voir qu'il s'est largement inspiré de l'ouvrage de Candolle, ou plutôt qu'il y a puisé une foule de documents utiles pour son exposition de l'origine des espèces. La part qu'Alphonse de Candolle a prise dans l'éla- 
boration de la théorie de la sélection naturelle devait être nécessairement faible. S'il admettait, comme l'une des idées fondamentales de sa géographie que les espèces se sont déplacées, peuplant les îles avant leur séparation du continent voisin, repeuplant les aires précédemment occupées par les glaciers, s'il acceptait aussi le fait, après avoir constaté que les espèces actuelles ne sont pas les mêmes que la majorité des espèces fossiles, qu'il pouvait y avoir eu création de nouveautés au dépends d'anciennes formes, il était bien loin d'imaginer la plasticité du monde organisé que Darwin allait révéler. C'est donc par l'extráordinaire richesse de ses analyses que Candolle a pu agir sur la pensée de Darwin, en lui fournissant en quelque sorte la matière, plus que des idées générales.

En revanche, on observera que peu à peu, ce sont les idées générales de Darwin qui pénétreront en Candolle et qui vont diriger ses recherches subséquentes. Ce fait apparaîtra clairement à qui prendre la peine de lire, après ces lettres, l'introduction d'Alphonse de Candolle à sa monographie des Smilacées.

\section{Candolle à Darwin}

Mon cher Monsieur,

Le 13 juin 1862

Je vous remercie extrêmement de m'avoir adressé votre article On the dimorphic condition of Primula ${ }^{4}$. Il est très curieux et bien propre à faire réflexion, comme tout ce que vous imprimez. J'ai engagé mon fils à en faire un extrait pour la Bibliothèque Universelle ${ }^{5}$ (Archives des Sciences), et il l'a rédigé effectivement pour un des prochains numéros. Le fait principal, celui de la fécondité supérieure par le croisement des deux formes les moins semblables, ne m'a paru se rattacher en physiologie qu'à un seul fait connu, celui de la fécondité supérieure et des produits plus vigoureux d'individus non parents les uns des autres, tandis que le breeding in and in est défavorable. Cela est bien mystérieux, en théorie on aurait plutôt supposé le contraire. Mais c'est un fait. Je n'ai pas vu si vous avez semé les graines obtenues par les divers

${ }^{4}$ On the two forms, or dimorphic condition, in the species of Primula and on their remarquable sexual relations, in J. Proc. Linnean Soc. 94 (1862).

5 Cas. de Candolle, in Arch. Sci. phys. nat., nouv. pér. 14 (1862) 192. Cet article n'est pas signé; on ne le trouve donc pas dans les listes bibliographiques de cet auteur et il a fallu l'indication contenue dans la lettre de son père pour le mettre au jour. 
croisements de vos Primula. On aimerait savoir si les deux formes reparaissent, indifféremment des graines de divers croisements, ou si telle catégorie de graines produit une des formes. Vous dites bien que le même pied conserve sa forme de fleurs d'année en année, mais qu'arrive-t-il de génération en génération?

La double forme des Borraginées m'avait frappé en les rédigeant pour le Prodromus. Je l'ai constaté dans cet ouvrage pour quelques espèces, par ex. l'Alkanna hispidissima DC. Prodr. X, p. 94, mais je n'avais point fait les recherches intéressantes que vous avez faites. On a aussi remarqué dans ... de la famille des Campanulacées des fleurs tardives ... presque nulle, qui sont bien fertiles, et dans deux espèces de fleurs, où les moins ... sont, autant qu'il m'en souvient, les plus fertiles, mais ce ne sont pas des faits ... analogues et l'on $n^{\prime}$ a pas expérimenté sur les $\ldots{ }^{6}$ Aurons-nous bientôt le grand ouvrage que vous annonciez comme donnant les preuves détaillées des faits dont vous parlez dans le volume sur l'origine des espèces? Je l'attends avec une vive impatience. En somme après vous avoir lu trois ou quatre fois, tantôt de suite, tantôt partiellement, je suis assez comme Asa Gray. J'aime votre théorie. Elle plait à mon esprit. C'est la seule qui rende compte de questions très obscures, inabordables par d'autres voies. Mais il lui faudrait des preuves, surtout quant à la natural selection. L'hypothèse générale d'une transmission indéfinie des formes au travers des siècles, avec des modifications plus ou moins graves, semble préférable à toute autre, mais que la natural Sélection soit le mode, voilà ce qui est vague dans mon esprit. Il y a tant de causes qui maintiennent longtemps les mêmes formes de génération en génération ou qui les ramènent! Il est si rare qu'une forme nouvelle paraisse, se conserve dans la protection de l'homme!

De ce dernier cas je ne connais aucun exemple constaté. Il y en a probablement, mais pas un seul n'a été prouvé à ma connaissance. Ces graves questions, sur lesquelles vous avez jeté un si grand jour, viennent de m'occuper à l'occasion d'une revue du genre Quercus ${ }^{7}$ pour le Prodrome et d'une analyse des travaux de Heer sur la Flore tertiaire que j'ai faite pour la Bibliothèque universelle de mai 1862.

${ }^{6}$ La copie, d'une main inconnue, sur laquelle nous travaillons, porte diverses corrections de la main d'A. DC. En marge du passage tronqué, transcrit ici, on lit, de son écriture bien reconnaissable: Plusieurs mots de ma lettre ont été détruits par enlèvement d'un morceau de papier.

${ }^{7}$ Cette partie du volume $(16,2)$ a paru en novembre 1864. Les réflexions auxquelles cette lettre fait allusion ont été publiées dans les Arch. Sci. phys. nat., nouv. pér. 15 (novembre 1862) 211. 
J'ai voulu traiter les Quercus ... étude sur l'espèce, au moyen de matériaux ... et aux formes. Les variations sur la ... sont étonnantes. Il en découle une quantité très grande de prétendues espèces des auteurs. Cela m'a conduit de nouveau à la question des origines des formes. J'ai ri de bon cœur en relisant la définition de Linné (Philos. bot. No. 157): Species tot numeramus quot diversae formae in principio sunt creatae. Combien le bonhomme croyait savoir de choses que nous ne savons pas cent ans après! Je n'ai découvert nulle part la date et le lieu précis d'origine d'un millier peut-être de formes des Quercus que je groupe en 200 espèces environ et que je réduirais encore si j'avais pour les formes étrangères autant d'échantillons que pour celles d'Europe et des Etats-Unis. Les formes principales du Quercus Robur $L$. (que je considère comme une espèce) sont antérieures à la séparation de l'Irlande d'avec la Grande Bretagne et par conséquent de celle-ci d'avec le Continent. Voilà bien du temps que les formes pedunculata et sessiliflora luttent dans toutes les forêts de toute l'Europe sans que l'une chasse l'autre. Les Américains sont moins acharnés. Si je me décide à publier ce que j’ai commencé de rédiger sur les Quercus, j'aurai l'honneur de vous le faire passer.

L'achèvement des Dicotylédones pour le Prodromus m'a occupé depuis quelque temps. J'espère ou plutôt je désire avoir assez de force pour reprendre la géographie botanique, lorsque j'aurai publié les volumes $X V$ et $X V I^{8}$ avec l'aide de divers collaborateurs. Malheureusement pour moi, les années avancent et les forces diminuent.

Agréez, mon cher Monsieur, l'assurance de ma haute considération et de tout mon dévouement.

Alph. De Candolle

Les recherches sur les Primevères n'ont cessé d'intéresser Darwin. Après cet article publié en 1862, il y revint à maintes reprises: en 1869 (On the character and hybrid-like nature of the offspring, in J. Linn. Soc. 10, 393), en 1876 (The effect of cross and self fertilisation in the vegetable kingdom, Murray, London), ailleurs encore. Il a répondu (dans le travail de 1869 et voir à ce sujet la lettre du 17 juin qui suit) à la question posée par Candolle en montrant qu'à la quatrième génération $\left(5^{\mathrm{e}}\right.$, en comptant les parents), le caractère de longistylie était transmis, presque intégralement.

L'allusion à Asa Gray (botaniste américain, correspondant assidu de Darwin) représente probablement une référence à une série d'articles d'Asa

${ }^{8}$ Prodr. vol. 15 (1864) 1; 15 (1862-66) 2; 16 (1869) 1. 
Gray parus dans l'Atlantic monthly en 1860 et réimprimés en 1861 sous le titre: A free examination of Darwin's treatise on the origin of species (notons au passage en sous-titre: Natural selection not inconsistent with natural theology!). Sans reprendre ici toute l'argumentation de l'auteur américain, argumentation très solide et parfois pleine d'humour, il faut noter cependant qu'il se prononce provisoirement pour la permanence probable de l'espèce. Mais, et c'est vraisemblablement le passage qu'Alphonse de Candolle a présent à l'esprit : [...] We adwise nobody to accept Darwin's [...] theory as true. The time has not come for that and perhaps never will. We also advise against a similar credulity on the other side, in a blind faith that species [...] "have no secondary cause". [...] An inevitable and legitimate hypothesis is on trial, $[. .$.$] a trial just now very useful to science ...$

C'est une position d'expectative favorable, celle même qu'adopte Alphonse de Candolle. Il attend, pour se prononcer, d'avoir reçu des preuves.

\section{Darwin à Candolle}

My dear Sir,

June 17th [1862]

I am extremely much obliged for your kind and very interesting letter. I am pleased that you are interested by the Primula case. Your questions and remarks show that you have gone to the root of the matter. I am now trying various analogous experiments on several plants and on the seedlings raised from the so-called heteromorphic and homomorphic unions; and the results (as far as I have yet seen, for the capsules are gathered, but not yet examined) are interesting. Whenever I publish, I will do myself the pleasure of sending you a copy. I am particularly obliged for your information on Alkanna. I have examined the small imperfect flower of Viola and Oxalis; the case is very different both functionally and structuraly from that of Primula. You kindly enquire about my large work; it does make progress but very slowly, owing to my own weak health and ill-health in my family. I have, also, been induced to publish a small work on the Fertilisation of Orchids ${ }^{9}$, which has taken up nearly ten months. As Mr. Bentham and Asa Gray think well of this Book, I have sent by this post a copy for you. One main object has been to show how wonderfully perfect the structure of plant is; another regards close breeding in and in, to which I see you have attended. I am not at all surprised that you are

${ }^{9}$ On the various contrivances by which British and foreign orchids are fertilized by insects and on the good effects of intercrossing, J. Murray, London 1862. 
not willing to admit natural selection; the subject hardly admits of direct proof or evidence. It will be believed in only by them who think that it connects and partly explains several large classes of plants; in the same way opticians admit the undulatory theory of light, though no one can prove the existence of ether or its undulations ${ }^{10}$. I hope you will publish on Quercus, and I shall be most grateful for a copy; the genus has long appeared to me preeminently interesting under the point of view to which you refer. I am, also, rejoiced to hear that you have the intention of again returning to Geographical Distribution. I believe few, or no one, can have read your truly great work with more care than I have, and no one can feel more respect and admiration for it and its author. Pray believe me, my dear Sir,

Yours sincerely and respectfully,

Ch. Darwin

Darwin a bien senti de la réticence dans l'attitude d'Alphonse de Candolle; la référence à l'article d'Asa Gray lui avait d'ailleurs fait comprendre que son correspondant genevois n'était pas encore parfaitement convaincu. L'admission candide: The subject hardly admits of direct proof or evidence montre bien que Darwin lui-même n'était pas satisfait de sa démonstration. En publiant en détail la masse incroyable de faits qu'il avait observés (et encore n'a-t-il pas tout publié!) il s'ingéniait à créer un climat, une opinion favorable à sa théorie indémontrable par une méthode directe. It will be believed in only by them who think ... révèle le fond de sa pensée; il faut croire à sa théorie puis admettre les démonstrations. Des preuves directes, il n'y en aura pas: la demande exprimée par Candolle restera sans réponse.

\section{Candolle à Darwin}

Le 18 septembre 1862

Mon cher Monsieur,

$J^{\prime}$ 'ai reçu et lu avec un grand intérêt le volume sur les Orchidées ${ }^{11}$ que vous avez bien voulu m'envoyer. Il est tellement rempli de faits qu'on voudrait

10 On trouve déjà cette même comparaison en 1860 dans une lettre de DARwin à AsA GrAY (Life and letters 2, 285): "Your distinction between an hypothesis and theory seems to be very ingenious; but I do not think it is ever followed. Every one now speaks of the undulatory theory of light; yet the ether is itself hypothetical, and the undulations are inferred only from explaining the phenomena of light.",

11 Voir ci-dessus, note ${ }^{9}$. 
pouvoir le lire à côté de chaque plante mentionnée pour comprendre mieux les détails, mais il y a souvent des idées générales qui se rattachent à l'ensemble de vos théories et c'est ce qui m'a le plus intéressé. Je viens de remettre au Directeur de la Bibliothèque Universelle pour la partie scientifique deux pages environ pour annoncer l'ouvrage ${ }^{12}$. Je n'ai pas osé entrer dans plus de détails, mais chaque lecteur doit être averti et voir le livre si cela l'intéresse. Les masses polliniques implantées sur les insectes ont donné lieu jadis à de singulières erreurs. Je ne sais plus quels botanistes les avaient prises pour des cryptogames! Wydler, autant qu'il m'en souvient, en a fait l'objet d'un article pour relever l'erreur, dans quelque journal allemand, il y a 25 ou 30 ans $^{13}$.

Les derniers cahiers du Bulletin de la Société botanique de France renferment des articles sur l'hybridation par Lecoq et sur le dimorphisme dans les Orchidées par Duchartre ${ }^{14}$ qui touchent un peu aux sujet dont vous vous occupez.

Je fais bien des vœux pour votre santé, car avec un si grand trésor accumulé de bonnes observations il serait fâcheux de ne pas continuer à publier. Mon travail sur les Quercus est fini ${ }^{15}$.

La partie descriptive dans le Prodrome sera la principale, mais je compte publier auparavant deux petits opuscules que j'aurai l'avantage de vous adresser. Agréez, je vous prie, en attendant, l'assurance de mes sentiments très dêvoués

Alph. de Candolle

La lettre qui suit (du 14 janvier 1863) est probablement la réponse. On a vu, en effet que Alphonse de Candolle termine la sienne en annonçant l'envoi de deux petits opuscules à paraître (sur le genre Quercus). Celui que Darwin a reçu doit être l'ouvrage mentionné à la note ?, et non la Note sur un nouveau caractère observé dans le fruit des Chênes parue à Genève, la même année.

12 L'article a paru sous le titre: Charles Darwin, On the various contrivances, etc. in Arch. Sci. phys. nat., nouv. pér. 15 (1862) 173. Il n'est pas mentionné par BRIQueT dans ses Biographies des botanistes à Genève.

13 Malgré tous nos efforts, nous n'avons pas réussi à découvrir à quoi A. DC. se réfère. WydLER ne semble pas avoir publié quelque chose sur les pollinies, bien qu'il se soit intéressé tôt aux Orchidées. En 1799 déjà, WÄchter (in Römer's Arch. Bot. 2, 2, 196) avait décrit - et compris - le mécanisme de la fécondation des Orchidées par les insectes.

${ }^{14}$ H. Lecoq, De la fécondation indirecte dans les végétaux, in Bull. Soc. bot. Fr. 9 (1862) 211. P. Duchartre, Note sur le polymorphisme de la fleur chez quelques orchidées, ibidem, 113. 15 Voir ci-dessus, note ?. 


\section{Darwin à Candolle}

My dear Sir,

$$
\text { Jan. 14th }[1863]^{16}
$$

I thank you most sincerely for sending me your memoir. I have read it with the liveliest interest, as is natural for me; but you have the art of making subjects, which might be dry, run easily. I have been fairly astonished at the amount of individual variability in the oaks. I never saw before the subject in any department of nature worked at so carefully. What labour it must have cost you! You spoke in one letter of advancing years; but I am very sure that no one would have suspected that you felt this. I have been interested with every part; though I am so unfortunate as to differ from most of my contemporaries in thinking that the vast continental extensions of Forbes, Heer and others are not only advanced without sufficient evidence, but are opposed to much weighty evidence.

You refer to my work in the kindest and most generous spirit. I am fully satisfied at the length in belief to which you go, and not at all surprised at the prudent reservations which you make. I remember well how many years ago it cost me to go sound from old beliefs. It is encouraging to me to observe that everyone who has gone an inch with me, after a period, goes a few more inches or even feet. But the great point, as it seems to me, is to give up the immutability of specific forms; as long as they are thought immutable, there can be no real progress in "epiontology" 17 and it matters very little to anyone except myself, whether I am a little more or less wrong on this or that point; in fact I am sure to be proved wrong in many points. But the subject will have, I am convinced, a grand future. Considering that birds are the most isolated group in the animal kingdom, what a splendid case is this Solenhofen bird-creature with its long tail and fingers to its wings! I have lately, been daily and hourly using and quoting your Geograph. Bot. in my book on "Variation under Domestication". With cordial thanks and sincere respect,

$$
\begin{aligned}
& \text { I remain, Dear Sir, } \\
& \text { Yours very faithfully, }
\end{aligned}
$$

I noted with delight case of Achenia.

Ch. Darwin

Il faudrait bien des pages pour étudier l'étonnant mémoire que Darwin venait de recevoir. Candolle y parvient, en effet, au prix d'une analyse 16 More letters 1, 233.

17 A. DC. est le créateur du mot. 
minutieuse de la variation, à une notion de l'espèce particulièrement claire et satisfaisante; mais ceci est en dehors du sujet. Ce qui nous intéresse, c'est l'écho de ses conversations épistolaires. Il admet maintenant - et nous suivons ici le développement de sa pensée scientifique - que les preuves n'existent pas toujours. Au moment même où Darwin le lui écrivait, Alphonse de Candolle publiait, dans des termes presques identiques la phrase révélatrice que voici: Il n'y a pas de démonstrations absolues en histoire naturelle. Toutes les assertions, toutes les opinions, ne sont que des probabilités fondées sur des indices, sur des faits susceptibles d'être mieux observés ou sur des chiffres de moyennes... (Arch. Sci.phys.nat. 15 [1862] 236).

Ce passage est à rapprocher d'un autre, bien significatif qu'Alphonse de Candolle écrivait en 1878 (cf. note ${ }^{39}$ ): Aussi quand je veux me représenter tel ou tel naturaliste moderne traduit devant une réunion de mathématiciens, de physiciens, de chimistes, etc., pour donner des preuves positives et directes de la transformation d'espèces végétales ou animales, assurément j'estime qu'il serait embarrassé. D'autre naturalistes, fidèles à d'anciennes idées, lui feraient une foule d'objections de détail, et le malheureux se verrait peut-être obligé d'avouer qu'il a des idées, mais pas de preuves absolument directes. Après cet aveu, il pourrait cependant réfléchir à la succession incontestable des flores et des faunes dans toutes les parties de la terre, et alors, il lui serait bien permis de dire à ses juges, en parlant de l'espèce «E pur si muove» (Arch.Sci.phys. nat. 61 [1878] 5).

Ainsi, les objections qu'il fait à Darwin, il se les fait à lui-même, tout seul. Et ne l'accusons pas de mauvaise foi: peu de savants ont été aussi honnêtes qu'Alphonse de Candolle. Mais on s'aperçoit que lui aussi doute, lui aussi a besoin de savoir que les autres, les Darwin par exemple, ne sont pas plus rassurés que lui-même lorsqu'il s'agit d'abandonner le terrain sûr de l'observation pour s'élancer dans l'incertitude des hypothèses.

(La réponse d'Alphonse de Candolle manque; elle a dû être très prompte puisque le 31 janvier, Darwin reprend la plume.)

\section{Darwin à Candolle}

My dear Sir,

Jan. 31 [1863]

I thank you for so kindly writing to me; and many parts of your letter have interested me much. I take a keen interest in progress of species-question, and 
am delighted to hear of the Count Saporta's views ${ }^{18}$. I really think that I believe in as much migration as even you believe in, and as shown in your admirable great work ${ }^{19}$; only $I$ do not believe nearly as much in continental extensions and I believe more (not very much more, I begin to supsect, and it pleases me greatly) than you do in modification in form. That is good remark on melons, and I shall probably use it, in conjunction with improvement of the pear. Also many thanks about oranges; I confess that I thought you rather too sceptical about Macfadyen's ${ }^{20}$ statements in W. Indies; I must change my tone.

What you say about antiquity of man, has occured to me; it must make considerable difference in our views on domesticated animals and plants. How very interesting the Swiss discoveries of the old Lake habitations have been; but I know only Rütimeyer's ${ }^{21}$ admirable essay, in which there is some little on Plants by Dr. Christ; I am very much obliged for your hint and must enquire; but I find German very slow reading and it wastes much time. I received a few days ago an address from New Zealand, and traces of Prehistoric man have been found there 22 !

I thank you for your Photograph, which I am glad to add to some others. I have never had a proper "carte photographique" taken of myself; but I enclose one $^{23}$ done 2 or 3 years ago by my son, if worth your acceptance.

18 C'est peut-être l'Examen analytique des flores tertiaires de Provence, paru à Zurich en 1861.

${ }^{19}$ Et ce que A. DC. croyait, à ce sujet, était peu de chose. Voir le ch. 8 de sa Géographie botanique et en rapprocher le passage suivant de son Etude sur l'Espèce (p. 333): «... les causes de transport par le vent, les courants et les oiseaux dont on parlait dans tous les traités de géographie botanique, n'ont pas agi une seule fois, d'une manière constatée, je ne dis pas pour transporter, mais du moins pour naturaliser une espèce phanérogame au-delà du plus petit bras de mer, à l'exception d'une ou deux espèces des sables maritimes $\gg$.

20 Le passage en question se trouve dans la Géographie botanique 2, 865. Dans une note malicieuse, A. DC. oppose Gallesio à MACFADYen citant leurs opinions parfaitement contradictoires sur l'hérédité chez les orangers. Il se ralliait à l'opinion du premier auteur qui était un spécialiste alors que le second ne l'était pas.

21 H. Christ, Bemerkungen über die vegetabilischen Reste der Pfahlbauten von Robenhausen, in L. Rütrmeyer, Die Fauna der Pfahlbauten der Schweiz (Neue Denkschr. Schweiz. Gesellsch. Naturf. 19 [1862] 224).

22 Address to the Philosophical Institute of Canterbury (N.Z.). The Report is given in the New Zealand Gov. Gazette, Prov. of Canterbury, Oct. 1862. (Note tirée de Life and letters $3,6$.

${ }^{23}$ Cette photographie, qui existe toujours et qui représente un Darwin visiblement fatigué, fait partie d'une série de portraits déposés au Conservatoire botanique, Genève, par la famille de Candolle. 
I am almost tired of my present big book ${ }^{24}$ (though a mere trifle to yours) on Variation under Domestication; for I have not sufficient knowledge to treat the plant-part well; but I have done so much that I will finish it.

Pray believe me, my dear Sir, with my best thanks and sincere respect,

$$
\text { Yours very faithfully, Ch. Darwin }
$$

L'ère des débats passionnés semble close; mais ce n'est qu'un répit momentané.

Darwin rassemble les documents pour un travail qu'il a en vue et demande l'aide de son correspondant genevois, mais, comme avant l'Origine des espèces, ce sont des matériaux dont il a besoin. Des idées, il en a en abondance, mais il lui faut les pièces justificatives.

\section{Darwin à Candolle}

My dear Sir,

Aug. 4th [1863]

Will you permit me to beg you to interest any young Botanist at Geneva to observe one point for me. But the possibility rests on Lythrum Thymifolia or Hyssopifolia growing near you and being still in flower. Vaucher says (Hist. Phys. Tome 2, p. 371) ${ }^{25}$ that these two species are dimorphic like Primula. $I$ have examined several dried specimens of L. Hyssopifolia and can see no trace of this fact. If either species present any difference in length of pistil and stamens, it is most likely that three forms should be presented. Could you get this point looked at for me; and if possible a few flowers of the two or three forms sent me in a letter. I may add that in some species of Lythrum two of the form have the pistil hidden within the calyx.

I have been trying most laborious experiments on $\mathrm{L}$. salicaria, and am extremely anxious to hear about and see if possible, L. thymifolia.

I trust to your kindness to forgive me for troubling you and remain, my dear Sir, with sincere respect,

Yours very faithfully,

Ch. Darwin

L'article parut dans J. Linn. Soc. Bot., 8: 169. 1865 et porte le titre: On the sexual relations of three forms of Lythrum salicaria. Comme pour

${ }^{24}$ The variation of animals and plants under domestication, 2 vol. Murray, London 1868.

${ }^{25}$ VAucher, Histoire physiologique des plantes d'Europe, 4 vol., Aurel, Paris 184.1. 
les Primevères, l'auteur cherche à définir le rôle de l'hétérostylie, mais il travaille ici sur des plantes trimorphes. Par des croisements répétés et l'examen des descendants, il montre que l'union légitime a lieu lorsque le pollen d'étamines longues est transporté sur un style long, le pollen d'étamines courtes sur un style court, le pollen d'étamines moyennes sur un style moyen: ce sont ces unions qui sont les plus fécondes. Pour les Lythrum thymifolia et hyssopifolia, Darwin a reçu des plantes sèches de Kew, de M. Hervett C. Watson, du Prof. Babington "and others". On veut espérer qu'Alphonse de Candolle est inclus dans cet "others".

(Les lettres suivantes manquent également. Nous n'avons plus rien pendant près de trois ans. Puis Alphonse de Candolle assiste, en 1866, au Congrès d'horticulture et de botanique de Londres, en qualité de président.)

\section{Candolle à Darwin}

Londres, 9 juin 1866

Mon cher Monsieur,

Je viens de passer 15 jours à Londres de la manière la plus agréable. Une chose cependant me fait de la peine, mais comme elle résulte seulement d'une erreur de ma part, je suis bien aise de vous l'expliquer. La première fois que j'ai assisté à une séance du comité relatif au Congrès botanique ${ }^{26}, j$ 'ai demandé de vos nouvelles et si nous aurions le plaisir de vous voir. Un de ces messieurs m'a répondu que vous étiez malade et il a ajouté (ou j’ai mal compris ce qu'il dit en anglais) que vous étiez absent. J'ai cru que vous étiez allé dans le Midi et avec beaucoup de regrets j'ai renoncé à l'idée de vous rencontrer. Ensuite, j'ai appris par M. Caspary et par Sir Charles Lyell que vous êtes à la campagne, mais c'était trop tard pour que je puisse aller vous présenter mes compliments. Permettez-moi de vous dire que c'est un véritable sujet de regrets pour moi, d'autant plus que nous allons nous diriger Madame de Candolle et moi, du côté d'Oxford et du Nord de la Grande Bretagne, de sorte qu'il ne sera pas possible de réparer ma négligence. Sir Charles Lyell et vous, n'avez peut-être pas une idée très exacte de ce que M. Lecoq a dit sur les plantes d'Auvergne et sur l'époque glaciaire. J'avais l'impression qu'il a surtout combattu l'idée d'un abaissement de température comme cause de l'extension des glaciers et qu'il a insisté sur la possibilité de grands glaciers quand il y a beaucoup d'humidité dans l'air. Je n'ai pas le souvenir qu'il ait

${ }^{26}$ Le Congrès a eu lieu à Londres du 22 au 31 mai 1866. A. DC. en était le président; il y fit un discours sur les rapports entre la botanique et l'horticulture. 
nié complètement l'époque glaciaire. Il a insisté sur la possibilité de transport de graines à de grandes distances par les vents, oiseaux etc., ce que j'ai examiné soigneusement, et je persiste pour mon compte, à croire qu'on a pris souvent des possibilités pour des réalités. L'observation m'a montré que dans 2 ou 3 siècles, dans les pays où l'on a le plus observé, aucune phanérogame ne s'est naturalisée autrement qu'à la suite d'une action directe ou indirecte de l'homme. Il faudra lire le discours improvisé de Lecoq, dans le Compte rendu ${ }^{27}$, pour s'en faire mieux l'idée.

M. Ruprecht a publié en allemand dans le Bulletin de l'Académie de StPétersbourg un mémoire curieux sur la terre noire du Midi de la Russie ${ }^{28}$. Il prouve par les débris végétaux et par l'absence de tout dépôt marin ou autre sur cette terre que c'était une île considérable remontant à l'époque tertiaire, où les graminées ont formé une énorme accumulation de détritus. Alors nous aurions là une source de nos végétaux d'Europe, avant et pendant l'époque glaciaire. J'ai fait traduire cet article pour la Bibliothèque Universelle de Genève, afin de pouvoir mieux le comprendre, car à première lecture d'un texte allemand assez mal rédigé, je ne puis pas apprécier bien l'opinion de l'auteur. J'ai lieu de croire M. Ruprecht un bon observateur et ce qu'il dit méritera votre attention ainsi que celle de Sir Charles et des autres géologues.

Agréez, mon cher Monsieur, l'assurance de mon dévouement très sincère et tous mes vœux pour une amélioration dans votre santé.

Alph. de Candolle

P.S. Mes respects, je vous prie, à Madame Charles Darwin.

LEcoQ (professeur à la faculté des Sciences de Clermont) avait cherché à expliquer à ses auditeurs le peuplement par les plantes d'un territoire bien délimité, l'Auvergne, dont les conditions physiques ont dû changer brusquement à la fin du tertiaire. La période glaciaire, pour Lecoq n'est pas le résultat d'un abaissement de la température mais au contraire d'un élèvement: On n'est jamais parvenu à créer de la vapeur sans chaleur, s'écrie-t-il,

${ }^{27}$ Le résumé a été publié dans Reports and proceedings of the international horticultural Exhibition and Botanical Congress, London 1866, 158-165, sous le titre: De la migration des plantes des montagnes.

${ }^{28}$ F.J. Ruprecht, Über die wissenschaftliche Bedeutung des Tschornosjom, in Bull. Acad. sci. (St-Petersbourg) 7 (1864) 425. Trad. fr. in Arch. Sci. phys. nat., nouv. pér. 26 (1866) 17, sous le titre: De l'importance scientifique du Tschornozeme ou terre noire. L'article original a été précédé d'un premier sur le même objet paru dans le vol. 7,418 , et suivi d'un troisième dans le vol. 9 (1866) 482. 
et comme toute la masse des glaciers a été suspendue dans l'atmosphère, il a fallu, lors de leur extension, une évaporation plus active, et un climat plus chaud pour produire de plus grands résultats.

Il semble que Lecoq admette que les hautes montagnes récemment soulevées aient servi de points de condensation pour les vapeurs atmosphériques, puisqu'il dit, un peu plus loin que nous ne pouvons ... accepter l'extension des glaciers au delà d'un certain rayon autour des montagnes. Alphonse de Candolle avait donc parfaitement rendu compte du discours entendu. Mais évidemment, la question qui l'intéresse plus directement est celle de la colonisation proprement dite, question liée naturellement à celle du transport des graines (voir, à ce sujet, la note infrapaginale ${ }^{19}$ ). Or Lecoq se place aux antipodes de Candolle. Ayant éliminé (on ne voit pas bien pourquoi) l'hypothèse d'une migration par voie de terre, il se trouve réduit aux voyages aériens: le vent et les oiseaux. Ce sont précisément les moyens qu'Alphonse de Candolle considérait comme peu efficaces, ou même comme inefficaces. L'ardent défenseur d'une théorie moderne qui prend assez exactement le contrepied du darwinisme, WILLIs (dans son monumental Birth and spread of plants, Genève 1949) arrive par des méthodes statistiques minutieuses aux mêmes conclusions que Candolle: l'eau, le vent, les oiseaux n'ont joué qu'un rôle insignifiant pour le transport des graines ou des fruits. Les flores se sont constituées par voie terrestre, tout au moins dans leurs traits essentiels et c'est sur place qu'elles se sont éventuellement diversifiées : produisant notamment la plupart des endémismes. On y retrouve donc l'écho des pensées d'Alphonse de Candolle, tandis qu'aux théories de Lecoqtout au moins en ce qui concerne l'origine des flores - on n'accorde aujourd'hui plus de crédit. (Cf. à ce sujet encore, Cain, S.A., Foundations of plant geography, 1944.)

Il est certain qu'au moment où il écrivait sa Géographie botanique, Alphonse de Candolle n'avait pas d'idées bien arrêtées sur l'origine de la flore alpine. Il savait (cf. op. cit. p. 1322) qu'elle représente un mélange, et il signalait en particulier que certaines plantes pouvaient être venues de Sibérie méridionale; les documents, cependant, lui manquaient encore, et l'on comprend l'intérêt très vif éveillé en lui par les travaux de Ruprecht. L'intrusion de plantes asiatiques dans la flore européenne à la suite du retrait des glaciers est un phénomène admis généralement les botanistes géographes qui se sont occupés de cette question; WulfF dans son Historical plant geography (trad. Brissenden, Waltham 1950) non seulement lui attribue une importance considérable mais suppose en outre que cette 
avance de la végétation orientale vers l'ouest n'est pas encore achevée actuellement (cf. op. cit. p. 157).

(Une lettre d'Alphonse de Candolle manque ici, et même probablement, plusieurs.)

\section{Darwin à Candolle}

My dear Sir,

July $6,1868^{29}$

I return you my sincere thanks for your long letter, which I consider a great compliment, and which is quite full of most interesting facts and views.

Your references and remarks will be of great use should a new edition of my book ${ }^{30}$ be demanded; but this is hardly probable, for the whole edition was sold within the first week, and another large edition immediately reprinted which I should think would supply the demand for ever. You ask me when I shall publish on the variation of species in a state of nature. I have had the M.S. for another volume almost ready during several years ${ }^{31}$, but I was so much fatigued by my last book that I determined to amuse myself by publishing a short essay on The Descent of Man ${ }^{32}$. I was partly led to do this by having been taunted that I concealed my views, but chiefly from the interest which I had long taken in the subject. Now this essay has branched out into some collateral subjects and I suppose will take me more than a year to complete. I shall then begin on species, but my health makes me a very slow workman. I hope that you will excuse these details, which I have given to show that you will have plenty of time to publish your views first, which will be a great advantage to me. Of all the curious facts which you mention in your letter I think that of the strong inheritance of the scalp-muscles has interested me most. I presume that you would not object to my giving this very curious case on your authority. As I believe all anatomists look at the scalp-muscles as remnant of the panniculus carnosus which is common to all the lower quadrupeds, I should look at the unusual development and inheritance of these muscles as probably a case of reversion. Your observation ${ }^{33}$ on so many remarkable

${ }^{29}$ Cette lettre a paru dans Life and letters 3, 99.

${ }^{30}$ Variation of Animals and Plants under domestication; la $1^{\text {re }}$ éd. a paru chez Murray, en 1868, la seconde, révisée, en 1875.

${ }^{31}$ Apparemment, ce manuscrit n'a jamais été publié.

32 The descent of Man, and Selection in relation to Sex, 2 vol. Murray, London 1871; la $2 \mathrm{e}$ éd., en un volume, date de 1874 .

${ }^{33}$ Ce doit être l'observation utilisée par A. DC. dans son Histoire des sciences et des savants, à la p. 333 de la $1^{\mathrm{re}}$ éd. (1873): «En général, le rôle qu'ont joué les batards ou des branches 
men in noble families having been illegitimate is extremely curious; and should I ever meet anyone capable of writing an essay on this subject I will mention your remark as a good suggestion.

Dr. Hooker has several times remarked to me that morals and politics would be very interesting if discussed like any branch of natural History and this is nearly to the same effect with your remarks. I agree almost entirely with what you say on acclimatisation and on graft-hybrids; I never was more perplexed in my life than to come to any probable decision about Cytisus adami. I suppose that you have seen the recent article in the Bot. Zeitung by Dr. Hildebrand ${ }^{34}$ on graft-hybrids in potatoes; this seems to me the best case yet recorded, and I am repeating his method of trial this year.

With respect to the hypothesis of Pangenesis ${ }^{35}$ very few persons approve of it, but is has some enthusiastic friends; nevertheless I am so presumptuous as to have much faith in its vitality. With cordial thanks for your great kindness and sincere respect, I remain, my dear Sir,

Yours very faithfully and obliged, Ch. Darwin

Il semble qu'Alphonse de Candolle n'ait jamais rien publié sur les muscles du scalp si développés dans sa famille. C'est Darwin qui s'en est chargé dans The descent of Man paru en 1871. A la page 20, il relate une observation qu'il doit à son correspondant genevois: $M$. de Candolle ... knows a

illégitimes de familles princières est remarquable, quand on pense à leur petit nombre. Je citerai: Dunois, le batard de Savoie, le prince Eugène, Vendôme, le connétable de Bourbon, Maurice de Saxe... Ces personnages... ont eu toujours de l'audace et presque toujours une absence complète de moralité. Ces deux traits de caractère, d'après le fait même de leur naissance illégitime, devaient se trouver chez les parents, du moins à l'époque de la transmission héréditaire» (même texte, p. 50 de l'éd. 1885).

${ }^{34}$ Einige Experimente und Beobachtungen 1) über den Einflu $\beta$ der Unterlage auf das Pfropfreis und 2) über den direkten Einflu $\beta$ des fremden Pollens ... in Bot. Z. 26 (1868) 321. Au sujet du Cytisus Adami, voir le travail de CASPary in Bull. Congrès int. Bot. et Hort. réuni à Amsterdam en 1865, Rotterdam 1866, où cet auteur propose l'explication admise aujourd'hui, d'une union tissulaire entre le Cytisus Laburnum et le C. purpureus, et lire surtout Darwin, Animals and plants under domestication, I, p. 387 et suivantes.

${ }^{35}$ Il existe un article de Darwin intitulé Pangenesis. Mais il n'a paru dans Nature qu'en 1871. L'hypothèse sur la Pangenèse est cependant bien antérieure à cet article. Dans une lettre à C. LyeLL (publiée dans Life and letters 3, 72), datée d'août 1867, l'auteur affirme qu'il a eu cette idée depuis 26 ou 27 ans, ce qui la ferait remonter aux environs de 1840. Voir encore ci-dessous la note 67 et lire les remarques d'A. DC. dans son Histoire des sciences et des savants, ed. 1, 464; ed. 2, 572. 
family, in which one member, the present head of a family, could, when a youth, pitch several heavy books from his head by the movement of the scalp alone ... His father, uncle, grandfather, and all his three children possess the same power to the same unusual degree ... This case offers a good illustration how persistently an absolutely useless faculty may be transmitted. (Voir plus loin des lettres du 20 juillet et du 8 mars 1871.) Il est évident que Darwin ne savait pas qu'il s'agissait d'une particularité de la famille de Candolle; il n'apprit ce détail qu'après la publication de son livre paru le 24 février 1871.

Le "short essay" mentionné dans cette lettre a pris, avec le temps, des dimensions considérables: deux volumes de plus de 400 pages chacun (voir ci-dessus, la note ${ }^{32}$ ) et il est peu d'ouvrages de Darwin qui ait soulevé, autant que celui-ci, l'intérêt passionné du public non scientifique, ce qui est assez compréhensible. L'Origine des espèces, la Fécondation des Orchidées, la Variation des animaux et des plantes étaient destinés aux naturalistes et on peut bien le dire sans offenser la mémoire de Darwin - aux naturalistes capables d'un effort de lecture prolongé. Mais sans doute, le public cultivé pressentait l'arrivée du livre; en 1860, Asa Gray avait déjà débattu la question (voir plus haut, après la lettre du 3 juin 1862), et en 1870, ne trouve-t-on pas, sous la plume du Rev. J.A. Picton, un essai intitulé: New Theories and the Old Faith? Darwin lui-même n'avait consacré que trois lignes dans l'Origine des espèces (p. 199) au problème de l'origine des races humaines, suivant en cela son idée, déjà exprimée en 1857 dans une lettre à Wallace: You ask whether I shall discuss "man". I think I shall avoid the whole subject, so surrounded with prejudices ... Puis, sous la pression des circonstances et surtout pour ne pas avoir l'air de se dérober, ainsi qu'il dit dans son Journal, il compose et publie la Descendance de l'Homme. Les attaques qui lui ont été prodiguées se sont bien atténuées mais - l'on ne nous contredira pas si nous rappelons ici certain procès américain - elles n'ont pas toutes cessé de nos jours. Darwin, pourtant, avait pris ses précautions, mais il est évident qu'il ne pouvait être compris, sur ce point particulier de l'évolution que par ceux qui avaient admis et adopté ses théories sur l'origine des espèces. Quant à la théorie de la Pangenèse, l'idée essentielle sur laquelle elle repose peut être réduite à quelques phrases: l'organisme tout entier se reproduit par chacune de ses particules. Par conséquent, chaque grain de pollen et chaque ovule consiste en une multitude de germes émanés de chaque particule. Cette théorie, ou plutôt cette hypothèse, est naturellement improuvable, mais elle paraissait à Darwin 
utile pour relier des faits aussi divers que la régénération de l'hydre mutilée par Trembley, la réapparition soudaine de caractères ancestraux, l'accumulation par l'usage et le non-usage. Darwin, par une sorte de divination, créait une sorte de théorie chromosomique avant la découverte des chromosomes, avant qu'on eût en main l'instrument magnifique forgé par Mendel et Naudin.

(La réponse d'Alphonse de Candolle manque; elle a dû suivre immédiatement la lettre de Darwin puisque la suivante est datée du 20 juillet.)

My dear Sir,

\section{Darwin à Candolle}

Freshwater, Isle of Wight, July 20 [1868]

I write a line merely to thank you for your kindness in telling me of your accidental error about the thorns, which I might have quoted and should probably have never observed the error.

I will be cautious in regard to the muscles of the scalp ${ }^{36}$, and will put the alternative of persistence. As these muscles are so highly variable, and are known in other to be apparently liable to tension, this view seemed to me the most probable in the very curious case which you have given me. My health has failed again in the usual manner, and I have been ordered to do no work, and so have come here for some weeks entire idleness, which I find very hard work. My wife is pleased to receive your kind remembrances. I do not think I expressed myself strongly enough, how much I was interested and pleased by your last long and very remarkable letter. Pray believe me with most sincere respect and thanks,

Yours very faithfully, Ch. Darwin

Contrairement à l'assertion de Francis Darwin qui prétendait que son père gardait toutes les lettres de ses correspondants (Life and letters, p. 119), beaucoup ont dû se perdre, à en juger par celles d'Alphonse de Candolle qui manquent à la série. Mais on sait aussi que Darwin avait l'habitude de communiquer à d'autres les lettres intéressantes (voir la preuve dans la lettre qui suit, et d'innombrables cas dans sa correspondance), ce qui explique certainement une partie des lacunes.

${ }^{36}$ Voir la lettre précédente. 


\section{Darwin à Candolle}

Oct. 11,1869

My dear Sir,

I am very much obliged for your extremely interesting letter. Your experiment $^{37}$ strikes me as a very valuable and simple one,- -so simple that now that it has been once suggested, I am surprized that no one ever thought of trying it before. Nägeli, indeed, in one of his recent essays complains that no one has made this experiment with Alpine \& Lowland forms of the same species. I forwarded your letter to Dr Hooker, who seems very glad to have the opportunity of making the trial. As I shall have many experiments in progress next spring, which cannot be deferred, and as I have little strength to spare, I believe it would be the wisest plan to send all the seeds to Kew. But I will open some of the packets and see how many seeds there are, so that if numerous I will try a few myself.

I will not however sow them till the spring, for I have lately had some experience in raising during the winter hardy annuals in my greenhouses and I find that they become much drawn up.

With my best thanks for your kindness and the most sincere espect, I remain, my dear Sir,

Yours very faithfully,

Charles Darwin

Si la remarque de Nägeli à laquelle Darwin fait allusion est bien celle reproduite ci-après, on ne peut qu'être étonné que l'auteur de l'Origine l'ait aperçue, noyée qu'elle est dans un texte qu'il avait d'ailleurs une peine énorme à déchiffrer, le dictionnaire à la main. Voici ce passage ${ }^{38}$ : Um die Natur einer Pflanzenform und die Grenzen ihrer Veränderlichkeit festzustellen, müssen vorzüglich auch Kulturversuche, welche sich an die Beobachtungen auf den natürlichen Standorten anschließen, gemacht werden ... Die Kultur einer Form aus anderen Gegenden, z.B. in den Alpen, wenn man deren Ur-

${ }^{37}$ Cf. A. DC., Tentatives d'expériences sur la question des modifications dans les espèces végétales à la suite d'un effet prolongé du climat, in Arch. Sci. phys. nat. 44 (1872) 105.

38 NäGELI ayant publié énormément vers 1866-1870, il est difficile de savoir à quel passage Darwin se réfère. Cependant, on trouve (dans Sitzber. k. b. Akad. Wiss. München, math.phys. Cl. 15 déc. 1866; réimpr. Bot. Mittheil. 3 [1881] 36) un article intitulé Über die Innovation bei den Hieracien und ihre systematische Bedeutung (cf. en particulier la note p. 46) qui paraît pourrait bien être celui auquel Darwin pensait et dont j'ai tiré la note reproduite ci-dessus. 
sprung genau kennt, hat mehr Werth als die Beobachtung auf 10 weitern natürlichen Standorten und diese kultivierten Exemplare verdienen eher eine Stelle im Herbarium als Exemplare von neuen, noch nicht vertretenen Localitäten. Il demande à maintes reprises, dans des textes de la même époque, qu'on examine les plantes en culture afin de découvrir, non seulement leurs caractères et la variabilité de ceux-ci, mais encore, leurs potentialités. Parmi les genres dont Nägeli s'occupait avec prédilection figure en première ligne le genre Hieracium; il s'agissait de faire dire aux "petites espèces» tout ce qu'elles cachent à celui qui ne les observe qu'en herbier ou dans la nature: il n'ouvrait pas la voie aux cultures expérimentales, mais il rejoignait Jordan (dont le Pugillus avait paru en 1864) qui exprimait au même moment des idées semblables.

Il ne semble pas que ces expériences aient eu une répercussion sur les travaux ultérieurs de Darwin; en tous cas nous n'avons pas pu découvrir, a u-delà de 1869, des échos de cette correspondance.

Par le détour de Nägeli et de Jordan ${ }^{39}$, nous arrivons de nouveau à Alphonse de Candolle ${ }^{40}$, qui pose (Tentative d'expériences, 108) le problème en ces termes: Mon but était de vérifier si des graines d'une même espèce, provenant de localités très éloignées, tout à fait différentes de climat, produiraient, semées dans une même localité, des individus ayant des conditions physiologiques différentes, par exemple ressentant d'une autre manière l'effet de la chaleur. Il est évident, d'après les textes qu'on possède, que l'auteur se proposait de faire observer la seconde génération dans différents jardins; il n'y a réussi qu'à Palerme, à cause du petit nombre d'espèces qui réunissaient les conditions exigées, et peut-être aussi à cause de l'inertie de ses correspondants. Dans le travail de 1878, on peut lire les conclusions auxquelles il était arrivé. [...] Il est visible:

1. Que des graines d'une même espèce venant de pays éloignés, semées les unes à côté des autres, sous les mêmes influences, ne donnent pas des individus qui végètent d'une manière absolument semblable.

2. Que dans certaines espèces, malgré la similitude des formes extérieures, la diversité de végétation suivant les origines est plus caractérisée que dans les autres ... Cependant, il faudrait savoir si des graines de plusieurs espèces

${ }^{39}$ Voir sur les travaux de Jordan, l'article de A. DC. dans Arch. Sci. phys. nat. 61 (1878) 260.

${ }^{40}$ De l'influence des climats sur les espèces végétales, dans Bull. Soc. bot. France 19 (1872) 177. Sur l'existence de races physiologiques dans les espèces végétales à l'état spontané, dans Arch. Sci. phys. nat. 61 (1878) 5. Cf. aussi, note ${ }^{37}$ ci-dessus. 
étant recueillies dans un même pays et semées les unes à côté des autres, leurs produits ne présenteraient pas quelquefois des diversités analogues de végétation.

Darwin ayant abandonné cet objet, la conversation, sur ce point, s'est arrêtée.

\section{Candolle à Darwin}

\section{Le 8 mars 1871}

Mon cher Monsieur,

J'ai commencé avec infiniment de plaisir la lecture de votre ouvrage sur l'Homme ${ }^{41}$ et ne veux pas tarder davantage à vous remercier de me l'avoir adressé. Si je différais, je serais trop tenté de vous envoyer des réflexions incidentes que chaque chapitre me fait naître, ce serait à n'en pas finir et vous me traiteriez de babillard.

$V_{\text {ogt }}{ }^{42}$ m'a abordé l'autre jour et m'a demandé quelle est la famille où le cuir chevelu se meut héréditairement depuis si longtemps. Pris à l'improviste, je n'ai pu que lever mon chapeau et lui montrer le fait sur l'original lui-même. Ayant trahi l'incognito avec lui, j'en fais autant avec vous, mais par égard pour mon cousin éloigné qui n'est point naturaliste, je vous prie de ne jamais publier le nom.

Le sens de l'odorat ${ }^{43}$ est bien plus inégal parmi les hommes civilisés que ceux de l'oü̈e ou de la vue.

Comme je l'ai fort développé, j'ai remarqué souvent la quantité d'individus des deux sexes qui en sont comme dépourvus, ce qu'on appelait en vieux français punais. S'il y a un individu Daltonien sur 20 ou 21, je crois que c'est davantage pour les odorats nuls ou indistincts et avec toutes sortes d'imperfections qui n'ont pas été constatées et classées. L'impression causée par les

41 Voir ci-dessus, note ${ }^{32}$.

42 Carl Vogt, appelé à l'Académie de Genève en 1852. Consulter, à ce sujet, l'Histoire de l'Université de Genève par Charles Borgeaud, dans le vol. consacré à l'Académie et

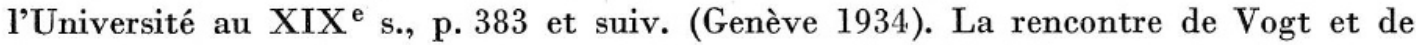
Candolle ne manque pas de piquant si l'on se rappelle qu'A. DC. avait abandonné son cours à la suite des événements politiques, au milieu du semestre d'hiver 1849-1850, et que le Conseil d'Etat, après avoir provisoirement confié sa chaire à MARC ThuRY, avait fait appel à Carl Vogt, précisément, pour le remplacer (Carl Vogt n'accepta d'ailleurs pas, et Thury continua le cours de botanique). Notons au passage que le livre de Darwin a paru le 24 février et que le 8 mars, Carl Vogt pouvait déjà en parler.

43 The descent of Man, p. 23. 
odeurs sur les chiens me parait singulière. J'ai un chien (bull-terrier) qui ne fait aucune attention aux odeurs végétales, même aux plus vives, comme la Rue, le Thym, le Céleri, tandis que la moindre odeur animale l'arrête et l'occupe un moment. Est-ce qu'il ne sent pas ce qui est végétal ou que l'idée d'un gibier étant liée à celle d'une odeur animale, il aime la retrouver? Je ne sais. Quant aux hommes il est certain qu'on pourrait beaucoup perfectionner ce sens, le seul pour lequel on n'ait rien fait de méthodique. Les parfumeurs lancent aux nez telle masse d'une odeur, c'est comme si les musiciens se contentaient de vous accabler d'un seul bruit ou les peintres de vous montrer une feuille d'une seule couleur. La succession de certaines odeurs et leur combinaison devraient avoir leur art, comme la musique et la peinture. Je suis persuadé que les odorats fins s'y plairaient. Du reste l'habitude de fumer éteint ce sens. La génération prochaine aura beaucoup moins besoin que nous d'être propre: elle s'apercevra moins de la saleté. $M$. Chevreul a conservé quoique octogénaire, une finesse extrême de vue (pour les nuances de couleur), de goût et d'odorat. Il estime le devoir beaucoup à ce qu'il n'a jamais fumé et n'a bu en général que de l'eau. Pour moi qui n'ai jamais pu supporter le tabac et qui pendant 29 ans n'ai bu d'ordinaire que de l'eau (crainte de la goutte paternelle) j'ai eu les mêmes avantages jusqu'à présent. La finesse du goût et de l'odorat est un bénéfice, malgré certaines privations ou désagréments dont on est frappé. Cela permet par exemple de fuir les émanations nuisibles de gaz, de miasmes d'égoûts ou d'hôpitaux, de refuser les viandes gâtées, etc. J'ai vu des ateliers d'ouvriers souffrir de ce que les chefs ne s'apercevaient pas d'un air vicié.

Les calamités de la guerre m'ont empêché de publier le $17^{\text {ème }}$ et dernier volume du Prodromus, dont les matériaux se préparent surtout en France. Heureusement, mes collaborateurs pourront encore consulter l'herbier du Muséum épargné par les bombes de Bismarck! Avec ce volume seront finies les Dicotylédones et je m'en tiens là ${ }^{44}$ ! C'est assez que deux générations successives se soient appliquées à une œuvre pareille, véritable série de monographies, dont les premières ont déjà beaucoup vieilli.

Profitant du loisir que cette interruption forcée me donnait, je me suis mis à rédiger des mélanges historiques et philosophiques, sur divers points des

${ }^{44}$ Le vol. 17 a paru le 16 octobre 1873 . Voir à la p. 304, dans le chapitre intitulé «Prodromi Historia, numeri, conclusio ", le passage suivant et qu'il faut rapprocher de celui de la lettre à Darwin: «Inde tempus amissum, inde volumina in sectiones divisa ... et tandem necessitas Prodromum ultra Dicotyledoneas non pergere et opus concludere, ne tertiam botanicorum generationem occideret!» 
sciences naturelles ${ }^{45}$. Un de mes articles devra probablement être supprimé d'après certains de vos chapitres sur l'homme ou s'il reste ce ne sera sûrement pas pour vous contredire, mais pour vous appuyer. Au surplus je n'ai aucun plan pour la publication. Il faut laisser les libraires rentrer dans leurs magasins et se reconnaître. Il faut donner au public français le temps de se remettre et voir si les allemands laisseront l'Europe en paix.

Recevez, mon cher Monsieur, l'assurance de mon ancien et sincère dévouement.

Alph. de Candolle

\section{Darwin à Candolle}

My dear Sir,

Nov. 2 [1872]

I am much obliged for your great kindness in having sent me your "Histoire des Sciences et des Savants" 46 . From what I see of the contents I look forward with real eagerness to read it; but I shall not be able to begin for a week or two. I have just published a small book on Expression ${ }^{47}$, which I did not suppose you would care to see; but from the nature of your recent studies, parts may possibly interest you, so I have told my Publishers to send you a copy. My health keeps very weak and I dread the exertion of grappling with the fearful subject of variation; so I am now employed in working up some observations in Botanical physiology made twelve years ago, which I intend publishing with some other new matters and some of my old papers on Climbing Plants, Dimorphism [etc? (petit mot illisible)] in one volume..$^{48}$

With cordial thanks and sincere respect, pray believe me

$$
\text { Yours very faithfully, }
$$

Charles Darwin

C'est dans l'ouvrage Expressions and emotions, au ch. 13 qu'on trouve les remarques auxquelles Alphonse de Candolle fait allusion dans sa lettre du

${ }^{45}$ Il annonce ici son Histoire des sciences et des savants qui devait paraître en 1873 et dont Darwin accuse réception dans la lettre suivante (2 nov.). Dans l'introduction à cet ouvrage, A. DC. l'appelle «un volume de mélanges».

${ }^{46}$ Histoire des sciences et des savants depuis deux siècles, suivie d'autres études sur des sujets scientifiques, en particulier sur la sélection dans l'espèce humaine, Genève 1873. La $2^{\mathrm{e}}$ éd. date de 1885 , mais on remarquera que la $1^{\text {re }}$ doit avoir paru en 1872, puisque Darwin en accuse réception en novembre de cette année-là.

47 The expression of the emotions in man and animals, Murray, London 1872.

${ }^{48} \mathrm{La}^{\mathrm{re}}$ édition a paru dans J. Linnean Soc., Bot. 9 (1867) 1 (read Feb. 2, 1865). La $2^{\mathrm{e}}$ éd. date de 1872; c'est celle-ci que mentionne Darwin. 
14 janvier et qu'on lira plus loin. Les principaux paragraphes sont intitulés: Nature of blush-Blushing in the various races of man-Confusion of mindCauses of blushing —Selfattention, the fundamental element-Shyness-Shame, from broken moral laws and conventional rules-etc.

La théorie de Darwin peut être résumée en peu de mots: L'homme (ainsi que la femme) porte un grand intérêt à son visage, il en soigne l'apparence. S'il a quelque raison de supposer que sa personne est critiquable aux yeux d'autrui, c'est à son visage qu'il pense en premier lieu, d'où une réaction du système vaso-moteur et des capillaires. Consciente d'abord, cette réaction est devenue, à l'usage et par hérédité, inconsciente. La rougeur subite n'indique donc pas la culpabilité, mais l'idée qu'on se fait que les autres nous croient coupables ${ }^{49}$.

\section{Darwin à Candolle}

My dear Sir,

Dec. $11,1872^{50}$

$I$ began reading your new book ${ }^{51}$ sooner that I intended, and when I once began, I could not stop; and now you must allow me to thank you for the very great pleasure which it has given me. I have hardly ever read any thing more original and interesting than your treatment of the causes which favour the development of scientific men. The whole was quite new to me, and most curious. When I began your essay I was afraid that you were going to attack the principle of inheritance in relation to mind; but I soon found myself fully content to follow you and accept your limitations. I have felt, of course, special interest in the latter part of your work; but there was here less novelty to me. In many parts you do me much honour, and everywhere more than justice. Authors generally like to hear what points most strike different readers; so I will mention that of your shorter essays, that on the future prevalence of languages ${ }^{52}$ and on vaccination ${ }^{53}$ interested me the most; as indeed did that

${ }^{49}$ Il n'est pas sans intérêt de rappeler ici le travail d'un botaniste lichenologue, C. MereJкоwsкy, sur l'Origine de la pudeur, publié dans Rev. anthrop. 29 (1919) 301.

${ }^{50}$ Cette lettre a été publiée dans Life and letters 3, 169.

${ }^{51}$ Voir ci-dessus, note ${ }^{46}$.

${ }^{52}$ Histoire des Sciences, ch. 4: En 1970, l'anglais sera parlé par 860 millions d'hommes, l'allemand par 124 et le français par $69 \frac{1}{2}$.

${ }^{53} \mathrm{Op}$. cit., ch. 6: Seuls les survivants à la petite vérole ont une descendance. Donc, naturellement, il se forme, en deux ou trois générations, une population mélangée de vrais ré- 
on statistics and free-will ${ }^{54}$. Great liability to certain diseases being probably liable to atavism is quite new idea to me. At p. 322 you suggest that a young swallow ought to be separated and then let loose in order to test the power of instinct; but nature annually performs this experiment, as old cuckoos migrate in England, and I presume elsewhere, some weeks before the young birds of the same year. By this way I have just used the forbidden word "nature", which after reading your essay I almost determined never to use again ${ }^{55}$. There are very few remarks in your book to which I demur; but when you back up Asa Gray in saying that all instincts are congenital habits ${ }^{56}, I$ must protest.

Finally, will you permit me to ask you a question; have you yourself, or someone who can be quite trusted, observed (p. 322) that the butterflies on the Alps are tamer than those on the Lowlands? Do they belong to the same species? Has this fact been observed with more than one species? Are they brightly coloured kinds? I am especially curious about their alighting on the brightly coloured parts of ladies' dresses, more especially because I have been more than once assured that butterflies like bright colours, for instance in India the scarlett leaves of Poinsettia.

Once again allow me to thank you for having sent me your work, and for the very unusual amount of pleasure which $I$ have received in reading it.

With much respect I remain, my dear Sir,

Yours very sincerely,

Charles Darwin

Darwin proteste à la fin du dernier paragraphe de sa lettre, quand il croit que Candolle soutient Asa Gray qui prétend que les instincts sont des habi-

fractaires et de non-contaminés: elle est prête pour une vague de recrudescence de la maladie. La pratique de la vaccination accentue la disposition normale d'une population à être moins affectée, mais elle la prépare mal à une nouvelle attaque de la maladie.

${ }^{54}$ Op. cit., ch. 6: La statistique et le libre arbitre. Les lois statistiques dérivent du libre arbitre; elles n'ont (contrairement aux lois civiles) aucune influence sur lui.

${ }^{55} \mathrm{Op}$. cit., ch. 7: Sur les différents sens du mot nature et par conséquent des mots naturel, surnaturel, etc., avec des citations tirées de Hooker, Darwin, Büchner, CL. Bernard, l'auteur fait ressortir la tendance qu'ont les hommes de science à accorder à la nature la faculté de vouloir, de s'efforcer, d'accorder, etc. Il propose de renoncer à l'emploi général du mot nature dans les ouvrages scientifiques et de ne le conserver que pour opposer la nature à l'art et pour désigner la nature d'une chose. Ce thème a été repris par lui dans sa Phytographie, p. 215.

${ }^{56}$ Op. cit., p. 321. L'auteur cite l'article d'Asa Gray paru dans Amer. J., sept. 1870: l'instinct n'est qu'une habitude héréditaire. Il ne semble pas que Darwin ait bien compris ce passage. 
tudes héréditaires. En réalité, Alphonse de Candolle interroge (l.c.,p. 321) ${ }^{57}$ : Quelle part faut-il attribuer ... à l'instinct, puisque l'instinct n'est qu'une habitude héréditaire? La réponse à cette question s'est trouvée difficile pour les animaux; elle l'est bien plus encore pour l'homme. On connaît la position prise par Darwin au sujet de l'instinct (cf. Origin of species, p. 207 et suiv.). On peut réformer une habitude, on ne peut pas lutter contre un instinct. Il ajoute (p. 242) cette phrase qui résume son opinion: ... It proves that with animals, as with plants, any amount of modification in structure can be effected by the accumulation of numerous, slight, and as we must call them accidental, variations, which are in any manner profitable, without exercise or habit having come into play. Darwin est positif là où Candolle en est encore à se poser des questions comme celles-ci (l.c., p. 322) : cette expérience est-elle accrue par hérédité ou par hérédité et sélection, chaque génération perdant de bonne heure les individus inaptes?

\section{Candolle à Darwin}

Mon cher Monsieur,

$$
\text { Le 14 janvier } 1873
$$

Vous avez été bien bon de me communiquer vos impressions sur la plupart des articles de mon dernier ouvrage et je vois fort heureusement qu'elles ont été approbatives. Comme nous cherchons tous les deux sincèrement et prudemment la vérité, je suis toujours heureux de me rencontrer avec vous. Si j'ai quelquefois restreint les conclusions auxquelles on semblait arriver par la variabilité et la sélection, je les ai aussi étendues dans d'autres cas, et j'estime être resté fidèle à vos principes en montrant, pour l'espèce humaine, que souvent le jeu des phénomènes produit des effets opposés qui se neutralisent plus ou moins et rendent les modifications extrêmement lentes ou peu importantes.

Le volume sur les Expressions ${ }^{58}$ que vous avez bien voulu m'adresser traite de sujets si nouveaux pour moi que j'avais tout à apprendre et n'ai rien à ajouter. Les détails sur les muscles de la figure sont une chose dont je ne m'étais jamais occupé et j'ai été surpris de la quantité de questions qui se rattachent au sujet.

Sur un point cependant, mes réflexions personnelles avaient devancé la lecture du livre, sans me conduire à aucune conclusion satisfaisante. J'ai

57 Voir ci-dessus, note ${ }^{46}$.

58 Voir ci-dessus, note ${ }^{47}$. 
souffert énormément jusqu'à l'âge de 55 ans de timidité (shyness). C'est au point qu'autrefois, à l'école, j'aimais mieux me faire renvoyer que d'avoir à me lever pour répondre en public et qu'ensuite dans plusieurs occasions j'ai fui devant des nominations, même par des moyens ridicules, tels que des propos déplaisants tenus sur des personnes influentes ou une exagération d'opposition qui n'était pas tout à fait vraie, pour éviter d'être nommé. Les examens me faisaient trembler. Un discours dans les Assemblées législatives dont j'étais membre et où j'étais assez bien vu, dépassait mes forces. Une présentation à un souverain me rendait muet. A 55 ans je suis rentré pour quelques années dans les Conseils de notre république, et il s'est trouvé, à ma grande surprise, qu'il ne m'en cô̂tait plus de prendre la parole. J'avais pensé d'après cela qu'un changement s'était fait dans mon système nerveux, par l'effet de l'âge. Il est vrai aussi que j'avais pu acquérir un sentiment plus juste de ma position à l'égard des autres. Après vous avoir lu, je me suis demandé si la crainte de montrer une figure ridicule devant le public avait été jadis la cause de mes faiblesses. Or, excepté entre 18 et 25 ans et dans les réunions où il y avait des dames, je ne crois pas que sela soit arrivé. Autour de moi on faisait peu d'attention à la figure, mais on vantait beaucoup l'esprit et les talents. J'ai pensé que mon malheur venait de l'hérédité. C'est de ma mère que je tenais ma disposition. Possible que dans sa famille on fût très vain de sa figure! Les gens timides pour paraître et parler sont quelquefois audacieux dans leurs idées et même dans leurs écrits. Ayant été dans ce cas il m'a paru qu'une cause aggravante de l'embarras est le contraste (dont on a la conscience) qui existe entre ses propres opinions et celles des autres. La timidité isole et l'isolement augmente l'indépendance des opinions. J.J. Rousseau, un des hommes les plus audacieux dans ses livres, était ridiculement timide. Lui, par exemple, tenait beaucoup à sa figure qui était agréable ${ }^{59}$.

Jusqu'à votre publication, je n'avais rien lu ou entendu de raisonnable sur cette question. Vous avez ouvert la voie, et surtout l'hérédité, ici comme

${ }^{59}$ A. DC. s'est fait portraiturer à de nombreuses reprises: on a de lui des bronzes, des peintures, des médailles, des gravures, des photos en grande quantité. Comme il était sans contredit un bel homme, j'avais cru pouvoir en tirer la conclusion qu'il se savait une figure agréable (cf. ma courte note sur Alphonse de Candolle dans Perspectives 9 [1953] 16). Son arrière petit-fils, M. Roger DE CANDolLe, est d'avis qu'il était conscient de sa valeur, de la place qu'il occuperait dans l'histoire des sciences, et qu'il ne voulait pas partager le sort de son illustre père dont on n'a que des portraits médiocres. On discerne clairement dans cette lettre que nous avions raison tous les deux: allusion d'une part à sa mère (pour l'admiration de soi) et d'autre part à Rousseau (pour la célébrité). 
ailleurs, explique beaucoup, mais il y a encore à perfectionner et compléter en suivant vos indications. M. Galton a été moins content que vous de ce que j'ai dit sur l'hérédité intellectuelle. Il m'a adressé une longue et amicale lettre d'observations ${ }^{60}$, à laquelle j'ai répondu dans le même esprit. Je crois voir entre nous des différences dans les conclusions et non des oppositions. La tendance générale de $M$. Galton et le titre de son livre l'indique ${ }^{61}$, est de montrer l'hérédité comme cause unique ou principale de la succession d'hommes distingués dans une famille. S'il parle des autres influences d'éducation, exemples, traditions, etc., c'est accessoirement. J'ai cherché à établir que ces autres causes sont souvent prépondérantes et me suis efforcé de préciser leur part dans plusieurs cas. Ainsi nous sommes d'accord quand il faut démontrer par des faits que l'hêrédité intellectuelle existe, mais nous différons dans l'estimation des résultats de cette cause combinée avec d'autres.

A vrai dire, je n'estime pas avoir été contraire à M. Galton, mais venant après lui, je crois avoir complété son travail en tirant de faits semblables des conclusions plus variées et plus complètes. Il se sera rendu, j'espère, à cette façon d'envisager nos travaux et il sait d'ailleurs à quel point j'ai rendu justice à ses recherches. Dans sa lettre, il me dit que les physiologistes anglais seront peu disposés à acquiescer à l'idée d'une influence de l'état momentané des parents sur les ovules et spermatozoaires au moment de la conception ou de la fécondation. Il me fait remarquer ce que je n'ignorais pas, qu'il s'écoule quelquefois plusieurs jours entre le détachement de l'ovule et sa fécondation. De là, sans doute, il faudrait conclure que l'état du mâle au moment essentiel aurait seul de l'importance, s'il s'agit d'un état physique ou moral tout à fait temporaire. Mais quand il y a une affection persistante pendant 8 jours, 15 jours, comme cela se voit, la femelle aussi pouvait modifier ses ovules.

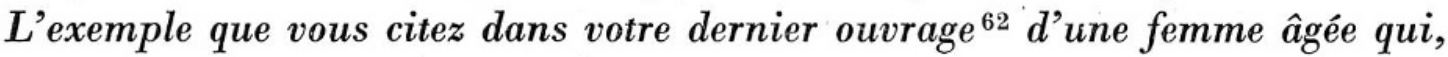
se croyant enceinte, avait vu le sang se porter dans des organes où il n'était plus en excès depuis longtemps, prouve bien l'effet d'une idée fixe sur les organes de la génération.

De là à supposer, par exemple, que la crainte excessive d'une nouvelle grossesse ou une antipathie de la femme contre celui dont elle doit subir l'autorité maritale peuvent altérer les ovules avant leur fécondation, il n'y a pas loin. Cette influence d'idées fixes ou de passions momentanées, comme la

\footnotetext{
60 Cette lettre n'a pas été conservée.

${ }^{61}$ Hereditary genius, Macmillan, London 1869.

62 Expression of the emotions, p. 341.
} 
colère, la peur, etc., est une hypothèse, déduite par analogie. Les faits connus sont principalement la lésion d'une chienne au moment de l'accouplement que j'ai citée d'après Lucas, et les idiots nés de parents en état d'ivresse ${ }^{63}$.

Depuis ma lettre à M. Galton, le hasard m'a fait connaître un cas bien frappant de cette dernière catégorie. On me l'a raconté à titre d'anecdote amusante et graveleuse; je l'ai remarqué comme un fait physiologique important. Voici :

J'ai connu à Genève un propriétaire, que j'appellerai $X$, qui est mort à plus de 80 ans, après avoir joui d'une excellente santé, dans une position aisée. Ses facultés intellectuelles étaient plutôt au-dessous de la moyenne, mais il a administré toujours sa fortune et s'est comporté comme un autre dans la vie ordinaire. Il était d'un degré de capacité qui lui aurait permis de siéger dans un conseil municipal ou un jury ordinaire ce qui lui est probablement arrivé. Son père et son frère étaient d'une intelligence un peu supérieure à la moyenne. Il avait épousé une femme intelligente, sans fortune, $q u i^{64} \ldots$

On doit regretter que la lettre de Galton manque à la collection, et surtout qu'Alphonse de Candolle n'ait pas gardé une copie de sa propre réponse, car, à n'en pas douter, le ton de ces lettres a dû être fort curieux. La phrase: Je crois voir entre nous des différences dans les conclusions et non des oppositions n'exprime certainement qu'une faible partie de la réalité. En fait, Galton doit avoir exaspéré Candolle qui était un statisticien, comme Galton, mais qui savait interpréter les statistiques avec une hauteur de vues, une absence de préjugés, une liberté que Galton était bien loin de posséder. On est confondu, en étudiant les résultats que ce dernier obtient, après avoir correctement posé le problème, de la somme incroyable d'idées préconçues qu'il parvient à tenir pour démontrées: la vérification statistique de l'inefficacité de la prière (qui ne se trouve pas dans Hereditary Genius mais dans Inquiries into human faculty) pour prolonger la vie des souverains est un défi au simple bon sens. Alphonse de Candolle qui en avait beaucoup n'a dû apprécier ni les prémisses ni les conclusions.

(Il est probable que la lettre qui suit est bien la réponse de Darwin à la lettre du 14 janvier 1873, mais la réponse à la question sur les papillons posée dans la lettre du 11 décembre doit s'être trouvée à la fin de la réponse d'Alphonse de Candolle, fin qui manque comme on a vu.)

${ }^{63}$ Histoire des sciences et des savants, ed. 1, p. 331.

${ }^{64}$ La copie de la lettre s'arrête ici, Alphonse de Candolle n'ayant pas reçu de M. Francis Darwin le dernier feuillet de sa lettre. 


\section{Darwin à Candolle}

Jan. $18[1873]^{65}$

My dear Sir,

It was very good of you to give up so much of your time to write to me your last interesting letter. Its evidence seems good about the tameness of the alpine Butterflies, and the fact seems to me very surprising; for each butterfly can hardly have acquired its experience during its own short life. Will you be so good as to thank Mr. Humbert ${ }^{66}$ for his note which I have been glad to read. I have received from a man, not a naturalist staying at Cannes, a similar account, but doubted about believing it. The case, however, does not answer my query, viz. whether Butterflies are attracted by bright colours, independently of the supposed presence of nectar. I must own that I have great difficulty in believing that any temporary condition of the parents can affect the offspring. If it lasts long enough to affect the health or structure of the parents, I can quite believe the offspring would be modified. But how mysterious a subject is that of generation!

Although my hypothesis of pangenesis has been reviled on all sides ${ }^{67}$, yet I must still look at generation under this point of view; and it makes me very averse to believe in an emotion having any effect on the offspring.

Allow me to add one word about blushing and shyness; I intended only to say that the habit was primordially acquired by attention to the face, and not that each shy man had attended to his personal appearence. With sincere thanks, believe me, yours very faithfully,

Ch. Darwin

Candolle à Darwin

Mon cher Monsieur,

Le 15 juillet 1875

Vous avez eu la bonté de m'envoyer votre volume intéressant sur les Plantes insectivores ${ }^{68}$. Je l'étudie avec plaisir après mon fils qui s'en est emparé le

65 More letters 1, 348.

${ }^{66}$ Aloïs Humbert, zoologiste et paléontologiste genevois, élève de F.J. Pıctet, avec lequel A. DC. était en correspondence (1829-1887).

${ }^{67}$ Voir, dans une lettre à Hooken (Life and letters 3, 78), les opinions des contemporains sur la Pangenèse. Cf. aussi note ${ }^{35}$.

${ }^{68}$ Insectivorous Plants, Murray, London 1875. 
premier et qui refait chez lui quelques-unes de vos expériences. Lorsque je décrivais les Sarraceniacées, pour le vol. XVII du Prodromus ${ }^{69}$, il m'est arrivé souvent de sortir des urnes une quantité de petits insectes et je m'étonnais de trouver leurs parties dures très bien conservées, le reste ayant disparu. Evidemment les substances liquides azotées ou autres avaient été absorbées, mais la plante se porterait peut-être aussi bien sans cela. C'est un fait à constater par la culture ou dans le pays. Je ne doute pas que vos découvertes ne conduisent les observateurs à d'autres dans le même genre, comme pour la fécondation par les insectes.

Le petit article sur les bourgeons de la même espèce provenant de deux localités, l'une plus au nord que l'autre, vous a été envoyé tel quel, c'est-à-dire un peu trop abrégé parce que l'Académie limite les publications dans les Comptes rendus à 6 pages ${ }^{70}$. Il faudra y revenir l'année prochaine avec de nouvelles expériences et avec des explications sur les autres méthodes qui conduisent aux mêmes conclusions.

Agréez, mon cher Monsieur, l'expression de mes remerciements et tous mes vœux pour la continuation de vos travaux.

Alph. de Candolle

Alphonse de Candolle, dans le "petit article», rend compte d'expériences récentes: Ayant obtenu d'un correspondant de Montpellier des rameaux de Charme, de Peuplier, de Tulipier et de Catalpa, il les mit én végétation à côté de rameaux prélevés à Genève sur des arbres de mêmes espèces. Les rameaux genevois se montrèrent - dans des conditions identiques - plus précoces que ceux de Montpellier. Il en tire, entre autres, une conclusion fort juste, celle de l'inégalité d'effet de la même température.

(Ici se place une lettre de Darwin à Casimir de Candolle sur les Dionaea, datée du 29 juin 1876 et qui est conservée dans les archives du Conservatoire botanique.)

69 Voir ci-dessus, note ${ }^{44}$.

${ }^{70}$ Des effets différents d'une même température sur une même espèce au nord et au midi, in C.R. Acad. sci. (Paris) 80, séance du 7 juin 1875. 


\section{Candolle à Darwin}

Le 16 décembre 1876

Mon cher Monsieur,

$J^{\prime}$ ai lu avec le plus vif intérêt l'ouvrage sur les Cross and self fertilizations ${ }^{71}$ que vous avez eu la bonté de m'envoyer. Le sujet est important, et comme méthode d'expérience, ou comme exposition, je ne crois pas que la physiologie végétale possède rien d'aussi satisfaisant. Votre persévérance pendant une série d'années aussi longue et l'ordre qui doit régner dans vos notes, me confondent ${ }^{72}$. Vos publications partielles avaient déjà fait connaître les résultats principaux, mais l'ensemble est ici très concluant et très curieux.

Malgré ce que vous dites que les conditions changent d'un emplacement à un autre dans un même jardin ou une même localité, il serait intéressant de comparer comme vous l'avez fait, les descendants d'une espèce prise dans des $\hat{\imath} l e s$, surtout de petites îles sans montagnes, avec les descendants de la même espèce venant d'un continent. Les individus d'une petite île sont presque complètement de la même famille, car les transports sont rares au travers de la mer, surtout ceux qui aboutissent à des plantes développées, portant des fleurs. A priori on peut soupçonner d'après vos observations, d'assez grandes différences entre les insulaires d'une espèce et les autres, mais on manque de comparaisons précises. Assurément les Iles de Ste Hélène, Maurice, Bourbon et Ceylan (quoique cette dernière soit rapprochée de l'Inde) ont été le théâtre d'invasions fréquentes d'espèces qui ont pris la place des anciennes espèces indigènes ou qui leur nuisent. Les espèces insulaires sont donc plus faibles, mais les individus d'une même espèce le sont-ils? C'est probable. Le degré relatif de faiblesse des individus insulaires indiquerait quelque chose sur l'ancienneté probable de l'isolement. Les formes extérieures sont peu modifiées dans beaucoup d'îles qu'on peut croire séparées depuis des milliers d'années, quoique géologiquement parlant, ce soit depuis des périodes peu anciennes. Ainsi les espèces des deux rives de la Méditerranée se retrouvent dans les îles intermédiaires et aux Canaries, à Madère, presque toujours sans qu'on hésite à

${ }^{71}$ The effects of cross and self fertilisation in the vegetable kingdom, Murray, London 1876. La $2^{\mathrm{e}}$ éd. est de 1878.

${ }^{72}$ Voir ci-dessous à ce sujet la lettre de Darwin du 28 mai 1880. Francis Darwin, dans Life and letters 1, 152, rappelle les méthodes de classement d'Alphonse de Candolle (décrites dans Mémoires et Souvenirs d'Aug.-Pyr. de Candolle, pièces justificatives, p. 493, et dans la Phytographie, p. 37), et les compare à celles de son père (Autobiography, in Life and letters 1,100 ) et dit le plaisir que les deux hommes ont éprouvé en découvrant qu'ils procédaient de semblables façons. 
les dire semblables. La séparation est cependant plus ancienne que celle de la Grande-Bretagne d'avec le Continent. L'avantage conféré par le mélange d'individus originaires de pays différents promet au Continent européen une superbe race à la suite des chemins de fer! Hélas, il n'est pas bien sûr que d'autres causes ne contrebalancent pas cet effet heureux du mélange des familles, l'indépendance personnelle favorisant aussi le vice et amenant une concurrence qui surexcite les facultés et crée des cas d'aliénations et de suicide.

J'ai lu avec plaisir l'opuscule de Mons. votre fils ${ }^{73}$ sur certaines glandes d'Acacia etc., aussi est-ce à lui que je prends la liberté d'adresser quelques lignes sur un point analogue.

Agréez, mon cher Monsieur, l'assurance de mes sentiments les plus dévoués.

Alph. de Candolle

C'est ici qu'on mesure peut-être avec le plus de précision, l'influence de Darwin sur Candolle: la distance est considérable entre le point où se trouvait ce dernier au moment où il écrivait sa Géographie botanique et le point où il se trouve maintenant. Il admet la concurrence des espèces entre elles (le "struggle for life"). Il fait même des hypothèses que Darwin n'a pas envisagées et qui ne seront pas retenues par la suite, mais il est indéniable qu'il pose les bases des travaux plus modernes de Guppy et de Willis (déjà cités) sur les espèces endémiques.

\section{Candolle à Darwin}

Janvier 1877

Mon cher Monsieur,

Permettez-moi de recommander à votre bienveillance et à celle de Monsieur Francis Darwin, mon fils Casimir, qui se trouve actuellement en Angleterre ${ }^{74}$ et qui me témoigne le désir bien naturel de vous être présenté. Il s'est occupé quelquefois des sujets dans lesquels vous avez ouvert la voie et assurément il apprendra beaucoup s'il a le bonheur de voir votre champ d'expérience et de causer avec vous. J'espère que cela pourra se réaliser sans vous faire perdre un temps excessivement précieux pour la science. Depuis ma lettre du mois

${ }^{73}$ On the glandular bodies on Acacia sphaerocephala and Cecropia peltata serving as food for ants, in J. Linn. Soc., Bot. 15 (1877) 388.

${ }^{74}$ Casimir de Candolle fit de très fréquents séjours en Angleterre; ses quatre enfants y sont nés. En 1876, il avait publié un travail Sur la structure et les mouvements du Dionaea muscipula au sujet duquel il a correspondu avec Darwin. 
de décembre, je vous ai adressé un opuscule sur un point relatif aux descriptions (la spire des feuilles, etc. ${ }^{75}$, en regrettant d'avoir si peu à vous offrir dans ce moment.

Toujours, mon cher Monsieur, votre très dévoué et affectionné.

Alph. de Candolle

\section{Candolle à Darwin}

Mon cher Monsieur,

Je vous remercie extrêmement de votre volume sur les Forms of flowers ${ }^{76}$. C'est un exposé très curieux de faits observés d'abord par vous et ensuite par d'autres, avec des comparaisons et réflexions d'un grand intérêt. La question de l'origine probable des plantes uni- et bisexuelles s'est présentée à moi en travaillant les Smilacées. Je regrette de n'avoir pas eu votre ouvrage avant l'impression des premières feuilles de notre volume de Monographies où j'en ai parlé. Quand vous recevrez ce volume ${ }^{77}$, en automne, vous verrez mes conjectures sur l'apparition et la dispersion géographique des trois genres de Smilacées proprement dites, l'un dioique, sans trace d'organes avortés (Heterosmilax), un second dioique, avec filets d'étamines stériles dans les fleurs femelles (Smilax), le 3ème hermaphrodite (Rhipogonum). Ce qui m'a fait incliner vers cette hypothèse (je ne l'ai pas expliqué expressément dans l'imprimé et j'ai eu tort), c'est que les feuilles se développent sur la plante dans cet ordre.

Le cotylédon et les premières feuilles de chaque rameau sont composés seulement de la gaine ou partie inférieure de la feuille; ensuite au milieu se développent les feuilles pourvues de limbe. Dans le bourgeon floral, c'est la même chose; les sépales et pétales sont des feuilles réduites à la base, et les pétales manquent même dans les Heterosmilax; enfin les feuilles centrales sont plus développées sous forme souvent d'étamines, pourvues d'anthères (limbe), mais pas toujours.

Dans le genre le plus développé, Rhipogonum, une espèce de la Nouvelle Zélande m'a offert quelque chose de bien rare, qu'on n'avait pas remarqué. La fleur n'est jamais fermée en bouton. Les plus jeunes montrent déjà des étamines

${ }^{75}$ Sur la désignation de la direction des spires dans les plantes, in Bull. Soc. bot. France 23 (1876) 192.

${ }^{76}$ The different forms of flowers on plants of the same species, Murray, London 1877; $2^{\mathrm{e}}$ éd. 1880.

${ }^{77}$ Les Monographiae Phanerogamarum, vol. I, ont paru en juin 1878. 
à découvert, sans préfloraison. C'est le contraire de la Cleistogamie ${ }^{78}$. Qu'en résulte-t-il pour la fécondation? Je ne sais. Les étamines des Rhipogonum, en particulier de celui-ci, contiennent peu de pollen. Le Rip. scandens est imparfaitement hermaphrodite, plus encore que les autres. Il est vrai qu'il se reproduit énormément par les pousses venant des rhizomes, comme c'est le cas de beaucoup de Smilax du Brésil, qui fleurissent rarement. Le pollen des Smilacées est hérissé de petites papilles, de sorte que la fécondation doit se faire plus par les insectes que par le vent ${ }^{79}$.

Dans les Rhipogonum les papilles sont très courtes, le pollen est presque volatil. Comme ils sont hermaphrodites, cela rentre dans vos idées. La fécondation se ferait d'une plante à l'autre malgré l'hermaphroditisme apparent, sans quoi (direz-vous) les produits auraient moins de vigueur.

En lisant votre page 11, je remarque que les Smilacées dioüques ne sont pas comme les Restiacées dont vous parlez. Les pieds mâles et femelles sont impossibles à distinguer, autrement que par les fleurs ou fruits, à moins que l'élévation (la taille) ne soit différente selon le sexe, ce qu'on ne voit pas dans les herbiers.

Plusieurs passages de votre dernier ouvrage et des précédents m'ont fait faire une recherche linguistique, d'après laquelle il paraît que les mots purpose, end, n'ont pas en anglais les sens précis que nous donnons en français aux mots but, fin. Je me disais en lisant au haut de la page 7 "their corollas have been increased in size for this special purpose", et p.9, vers le bas, "subserve any special end". Mr. Darwin, dont l'esprit philosophique poursuit méthodiquement les causes et les effets, aurait mieux fait de dire; their corollas being increased in size the consequence is, etc. et p. 9 au lieu de subserve any special end ... have any effect, car l'observation ne montre que des formes et des conséquences ou effets, non des buts ou intentions. Nos mots but, fin, supposent une intention, une volonté extérieure; or pour connaître une intention, il faut questionner celui auquel on l'attribue ou l'entendre s'expliquer, ce qui n'arrive pas dans les phénomènes naturels. Que diraient des français ou des

${ }^{78}$ Ce même phénomène a été redécrit par nous sans savoir qu'Alphonse de Candolle l'avait déjà vu, à propos d'une plante d'Australie, l'Anthocercis littorea; le nom de chasmostegie a été proposé (cf. Сн. Baenni, Organogénie de la fleur chez l'Anthocercis littorea, in Bull. Soc. bot. suisse 54 [1944] 640, et l'Ouverture du bouton chez les fleurs de Solanées, in Candollea 10 [1946] 399). On notera qu'il a donc été observé une première fois sur une plante australienne, et une deuxième fois sur une plante de Nouvelle-Zélande, dans deux groupes (Smilacées et Solanées) fort éloignés l'un de l'autre.

${ }^{79} \mathrm{Il}$ ne semble pas que cette opinion puisse encore être défendue. Cf. Erdtuan, Pollen morphology and plant taxonomy, Almqvist \& Wiksell, Stockholm 1952. 
hollandais s'ils lisaient dans un livre de géologie ; «à la fin de l'époque tertiaire la vallée de la Tamise s'est abaissée dans le but que Londres eût un commerce immense etc.» ou «les côtes de France se sont élevées dans le but que les Français ne fussent pas navigateurs». Ils diraient assurément: qu'en sait-on? L'auteur a pris des conséquences pour des buts, des effets pour des causes. Mais ces doutes et ces critiques de mon esprit sont tombés en ouvrant mon dictionnaire anglo-français de Spiers: «Purpose. But, fin, effet, intention, dessein, usage. End. Bout, extrémitê, fin, but, objet, cause».

Ainsi, chacun de ces mots anglais a deux sens contradictoires, tantôt un but prémédité, tantôt un effet, tantôt une cause, tantôt un résultat. Je doute que vos traducteurs aient fait attention à cette difficulté et je crains que l'ambiguité de ces mots anglais ne conserve dans le public anglais une certaine confusion d'idées peu philosophique. En tous cas, si je vous ai critiqué d'abord in petto, c'était ma faute; je n'avais pas compris le vague inévitable déterminé par la langue ${ }^{80}$.

Mon fils, qui compte comme un de ses bons jours, celui que vous avez bien voulu lui accorder, se rappelle à votre bon souvenir.

Dites, je vous prie, à Mr. Francis Darwin que Mr. Delpino a été plus heureux que nous en observant des Smilax ${ }^{81}$. Je soupçonnais une excrétion de l'extrémité des feuilles et nous ne l'avions pas vue, moi à Genève sur le Smilax excelsa, et Mr. Francis à Kew sur des Smilax probablement en serre, mais les pieds observés n'étaient pas dans des conditions ordinaires de climat et végétation. A Gênes, Mr. Delpino a vu, sur deux espèces (S. aspera et bonanox) une matière sucrée produite par le tissu glandulaire qui se trouve au bout de la feuille, du côté extérieur, surtout dans les jeunes feuilles des pousses annuelles. Cette matière sucrée est recherchée par les fourmis. D'après les échantillons d'herbiers, la sécrétion doit être plus forte dans certaines espèces de Chine, du Brésil, etc. Delpino, tout pénétré des anciennes théories sur les causes finales, cherche le but, l'intention, pour lesquelles la sécrétion et l'abord des fourmis existent dans ce cas.

Il croit, en général, que les fourmis arrivent afin de débarrasser les arbres des larves d'insectes nuisibles. J'aimerais mieux l'hypothèse que les matières sécrétées ont pour but de nourrir les fourmis. Les jardiniers ont une troisième manière de voir qui est de considérer les fourmis comme un fléau, dont il faut

${ }^{80}$ Voir ci-dessous l'opinion de Darwin (lettre du 24 janvier 1881). A. DC. a traité ce sujet dans sa Phytographie, p. 212.

${ }^{81}$ La correspondance de DeLpino où ces faits sont relatés se trouve dans les archives du Conservatoire botanique, Genève. 
se débarrasser le plus possible. Enfin, je préférerais aux hypothèses une bonne expérience; dans une rangée de cerisiers ou autre arbre fréquenté par les fourmis, enduire quelques tronc de coaltar de manière à empêcher la visite de ces insectes, et voir si la végétation, floraison et fructification seraient différentes sur les arbres avec et sans fourmis. On saurait alors les conséquences, les effets, au lieu de faire des suppositions de toute nature sur des intentions ${ }^{82}$ !

Je me suis laissé entraîner au plaisir de causer avec vous. Il ne me reste qu'à vous prier de m'excuser de la longueur et d'agréer, mon cher Monsieur, l'assurance de tout mon dévouement.

Alph. de Candolle

Plus encore que précédemment, on voit ici s'affirmer l'intérêt que prenait Alphonse de Candolle pour les idées darwiniennes. Mais il faut lire le paragraphe 9 des Smilacées pour s'en convaincre parfaitement: L'histoire d'un groupe de végétaux ou d'animaux se compose de ses migrations successives ou extinction dans différentes régions du globe, et des changements de forme ou de propriétés physiologiques des individus, pendant une série immense de siècles ... En ce qui concerne les Smilacées proprement dites, on peut affirmer, avec peu de chances d'erreur, qu'elles sont au nombre des plus anciennes formes de plantes phanérogames ... Une longue hérédité paraît avoir fixé leurs formes ...

Et pourtant: Les fossiles que l'on connaît ne confirment pas encore cette ancienneté présumée.

C'est donc qu'Alphonse de Candolle, conquis par les idées nouvelles, étaie son raisonnement sur la dispersion géographique, l'analyse des documents morphologiques et il exprime pour finir son opinion sur l'évolution du groupe dans le passé. Quel hommage rendu à Darwin!

\section{Darwin à Candolle}

My dear Sir,

Aug. 3, $1877^{83}$

I must have the pleasure of thanking you for your long and interesting letter. The cause and means of the transition from a hermaphrodite to an unisexual condition seems to me a very perplexing problem, and I shall be extremely glad to read your remarks on Smilax, whenever I receive the essay ${ }^{84}$ which you

${ }^{82}$ Il n'y a pas de traces, dans les travaux de Darwin ou des Candolle (postérieurs à cette lettre) qui indiqueraient que ces expériences aient été entreprises.

${ }^{83}$ More letters 1, 368.

${ }^{84}$ Voir ci-dessus, note ${ }^{77}$. 
kindly say that you will send me. There is much justice in your criticisms on my use of the terms object, end, purpose; but those who believe that organs have been gradually modified by natural selection for a special purpose, may I think use the above terms correctly though no conscious being has intervened. I have found much difficulty in my occasional attempts to avoid these terms; but I might perhaps have always spoken of a beneficial or serviceable effect. My son Francis will be interested by hearing about Smilax. He has dispatched to you a copy of his paper on the glands of Dipsacus ${ }^{85}$, and I hope that you will find time to read it, for the case seems to me a new and highly remarkable one. We are now hard at work on an attempt to make out the function or use the bloom or waxy secretion on the leaves and fruit of many plants; but I doubt greatly whether our experiments will tell us much. If you have any decided opinion whether plants with conspicuously glaucous leaves are more frequent in hot than in temperate or cold, in dry than in damp countries, I should be grateful if you would add to your many kindnesses by informing me. Pray give my kind remembrances to your son, and tell him that my son has been trying on a large scale the effects of feeding Drosera with meat, and the results ${ }^{86}$ are most striking and far more favourable than I anticipated.

With the highest respect,

I remain, my dear Sir,

Yours sincerely,

Charles Darwin

\section{Darwin à Candolle}

My dear Sir,

Oct. 10,1877

I thank you cordially for your two letters on bloom ${ }^{87}$ which will be very useful to.us as showing how much or how little is known about the distribution of plants thus provided. Your remarks seem very just with respect to evaporation and absorption. On the latter head we have made very few experiments as yet

85 On the protrusion of protoplasmic filaments from the glandular hairs of the common teasel (Dipsacus sylvestris), in Quarterly J. micr. sci., new ser. 17 (1877) 245.

86 Voir ci-dessus, note ${ }^{74}$. Les observations de F. Darwin ont été publiées dans le J. Linn. Soc., Bot. 17 (1878) 17.

87 Voir la lettre précédente. A maintes reprises, Darwin est revenu sur cet objet, la signification de la pruine: dans ses lettres à Hooker, Farrer, Asa Gray, Thiselton Dyer, F. von Mueller (cf. Life and letters 3, 339 et More letters 2, 409-10). 
the investigation has been a tedious and difficult one, but I hope that we shall be able to throw some little light on the subject. Again accept my thanks for your great kindness, and I remain, with much respect,

$$
\begin{aligned}
& \text { My dear Sir, } \\
& \text { Yours sincerely, } \\
& \text { Charles Darwin }
\end{aligned}
$$

\section{Candolle à Darwin}

Samaden (Engadine), 10 Août 1898

Mon cher Monsieur,

Les nouvelles arrivent tard et incomplètement dans l'extrémité orientale de la Suisse et à 1724 mètres d'élévation, à douze heures des chemins de fer; cependant un journal m'a appris votre nomination par l'Académie des Sciences de l'Institut, comme correspondant ${ }^{88}$. Permettez-moi de vous dire à quel degré j'en ai été satisfait. Chose singulière! Dans le cas actuel ce n'est pas l'élu qu'il faut le plus féliciter, car votre position est si élevée dans la Science qu'un titre de plus est assez indifférent. Mais c'est l'Académie à laquelle je m'intéresse et qui tardait beaucoup trop à reconnaître vos mérites. Le système de présentation par sections vous a nui parce qu'on vous renvoyait de la zoologie à la botanique ou vice versa. Malheureusement, il y avait aussi des préventions ridicules pour des motifs non scientifiques ${ }^{89}$. J'ai craint quelquefois qu'on ne vous traitât comme jadis le Dr Priestley qui avait découvert l'oxygène - c'est vrai-mais qui était républicain, de sorte que les Académies royales de Paris et de Berlin ne le voulaient pas sur leurs listes. Enfin, vous avez survécu aux objections fausses et hors de place qu'on suscitait contre vous! Elles ne venaient pas des membres de l'Académie que je connais le plus et auxquels je parlais souvent de vous, mais plutôt de mathématiciens, physiciens, etc., avec lesquels j'ai moins de rapports. Même $M$. de Quatrefages ${ }^{90}$, qui combat vos opinions en histoire naturelle, m'a dit avoir voté pour vous dans une autre occasion où la discussion

${ }^{88}$ Cet honneur fut attribué en même temps à AsA GrAY. Dans une lettre à ce dernier, Darwin s'amuse gentiment du titre nouveau qu'ils ont reçu et constate qu'il a été élu dans la section botanique, lui qui connaît juste assez les plantes pour classer les pâquerettes dans les Composées et les pois dans les Légumineuses.

89 Théologiques!

90 Non seulement Quatrefages vota pour Darwin (bien qu'ils fussent d'opinions opposées sur l'origine des espèces), mais il publia à la mort du savant anglais un magnifique hommage, nuancé, très digne (C.R. Acad. Sci. Paris 114, séance du $1^{\mathrm{er}}$ mai 1882). 
sur votre compte fut très vive. La jeunesse française vous est plus favorable, surtout en province, et le renouvellement du personnel de l'Académie vous aurait fait nommer tôt ou tard, mais il vaut mieux que cela soit arrivé maintenant.

Vous aurez reçu le $1^{\text {er }}$ volume de nos Monographiae Phanerogamarum ${ }^{91}$ que j'ai dit au libraire de vous envoyer. C'est un livre à consulter dans l'occasion, voilà tout. A peine une page ou deux dans les généralités sur les Smilax valent-elles la peine d'attirer votre regard. Par parenthèse en comparent les Smilax fossiles avec les vivants, j'ai pris une triste opinion des prétendues espèces végétales fossiles. Les caractères génériques ne peuvent pas du tout être devinés par les feuilles qui sont ici. La seule chose connue, et encore, les feuilles sont réduites à des limbes sans pétioles qui ne permettent pas de distinguer des espèces. Je n'ai jamais vu une donnée paléontologique aussi vague.

Mes compliments à Messieurs vos fils. Je voudrais les rencontrer une fois dans cette curieuse vallée de l'Engadine où les courses de montagne ont un attrait tout particulier, surtout pour la jeunesse.

Agréez, je vous prie, mon cher Monsieur, l'assurance de mes sentiments les plus dévoués.

$$
\text { Alph. de Candolle }
$$

Darwin à Candolle

My dear Sir,

Aug. 15, 1878

I thank you cordially for your most kind letter, which has told me much that was new and has interested me much. I cannot pretend that I care greatly about my election to the Institut, but I do care very greatly for the kind sympathy of my friends, and for none more than for yours.

I have been away from home for a fortnight and shall not return for another week; and when there I shall no doubt find the book which you have been so kind as to order to be sent me, and will look to Smilax and any other generalities.

I am always sorry to hear about the insecurity of the identification of fossile leaves; but Hooker has frequently made with vehemence nearly the same remarks as yours.

With the greatest respect, believe me

Yours truly obliged

Charles Darwin

${ }^{91}$ Voir ci-dessus, note ${ }^{77}$. 
P.S. If your son is with you pray tell him that I hear that Du Barry ${ }^{92}$ has cultivated Utricularia with and without aquatic animals, and that the former or fed plant have flourished in a stupendous manner.

\section{Darwin à Candolle}

My dear Sir,

[May] 28th $1880^{93}$

I am particularly obliged to you for having so kindley sent me your Phytographie ${ }^{94}$; for if I had merely seen it advertised, I should not have supposed that it could have concerned me. As it is, I have read with very great interest about a quarter, but will not delay longer thanking you. All that you say seems to me very clear and convincing, and as in all your writings I find a large number of philosophical remarks new to me, and no doubt shall find many more. They have recalled many a puzzle through which I passed when monographing the Cirripedia; and your book in those days would have been quite invaluable to me. It has pleased me to find that I have always followed your plan of making notes on separate pieces of paper: I keep several scores of large portfolios, arranged on very thin shelves about 2 inches apart, fastened to the walls of my study, and each shelf has its proper name or title; and I can thus put at once every memorandum into its proper place ${ }^{95}$. Your book will, I am sure, be very useful to many young students, and I shall beg my son Francis, (who intends to devote himself to the physiology of plants) to read it carefully.

As for myself I am taking a fortnight's rest after sending a pile of M.S. to the Printers, and it was a piece of good fortune that your book arrived as I was getting into my carriage, for I wanted something to read whilst away from home. My M.S. relates to the movements of Plants ${ }^{96}$, and I think that $I$ have succeeded in showing that all the more important great classes of movements are due to the modification of a kind of movement common to all parts of all plants from their earliest youth.

92 Inconnu. S'agirait-il de A. DE BARY? Pourtant, à notre connaissance, cet auteur n'a rien publié sur ce sujet.

${ }^{93}$ Lettre publiée dans Life and letters 3, 332.

${ }^{94}$ La Phytographie, ou l'art de décrire les végétaux considérés sous différents points de vue, Masson, Paris 1880.

${ }^{95}$ Voir ci-dessus, note ${ }^{72}$.

${ }^{96}$ Darwin annonce ici son dernier grand ouvrage: The power of movement in plants, Murray, London 1880. 
Pray give my kind remembrances to your son and with my highest respect and best thanks, believe me

\author{
My dear Sir, \\ Yours very sincerely, \\ Charles Darwin
}

P.S. It always pleases me to exalt Plants in the organic scale, and if you will take the trouble to read my last chapter, when my book (which will be sadly too big) is published and sent to you, I hope and think that you also will admire some of the beautiful adaptations by which seedling plants are enabled to perform their proper functions ${ }^{97}$.

En automne de cette même année, Alphonse de Candolle rendit visite à Darwin dans sa maison de Down; il a raconté cette journée, dans un opuscule maintes fois mentionné, Darwin considéré au point de vue des causes de son succès. L'impression est mélancolique: La serre dans laquelle de si belles expériences ont été faites ne contenait plus qu'un cep de vigne. La santé de Darwin n'avait jamais été excellente, mais depuis quelque temps, elle donnait des inquiétudes: son activité scientifique s'était considérablement ralentie; il prenait des vacances.

\title{
Darwin à Candolle
}

My dear Monsieur de Candolle,

Jan. 24, $81^{98}$

It was extremely kind of you to write me so long and valuable a letter, the whole of which deserves careful consideration. I have been particularly pleased at what you say about the new terms used, because I have often been annoyed at the multitude of new terms lately invented in all branches of Biology in Germany; and I doubted much whether I was not quite as great a sinner as those whom I have blamed. When I read your remarks on the word "purpose" in your "Phytographie", I vowed that I would not use it again ${ }^{99}$; but it is not easy to cure oneself of a vicious habit. It is also difficult for any one who tries to make out the use of a structure to avoid the word purpose. I see that I have probably gone beyond my depth in discussing plurifoliate and unifoliate leaves ;

${ }^{97}$ Le chap. est intitulé: Summary and concluding remarks. C'est là qu'on trouve l'expression: ... The tip of the radicle ... acts like the brain ...

98 More letters 2, 429.

${ }^{99}$ On se rappelle que dans sa lettre datée du 11 décembre 1872 , il avait annoncé qu'il n'emploierait plus le mot nature; ici, il écoute de nouveau Candolle au sujet du mot purpose (voir la lettre du 31 juillet 1877 et la note ${ }^{80}$ ). 
but in such a case as that of Mimosa albida, where rudiments of additional leaflets are present we must believe that they were well developed in the progenitor of the plant. So again when the first true leaf differs widely in shape from the older leaves, and resembles the older leaves in allied species, is it not the most simple explanation that such leaves have retained their ancient character, as in the case of the embryos of so many animals? 100

Your suggestion of examining the movements of vertical leaves with an equal number of stomata on both sides, with reference to the light, seems to me an excellent one and I hope that my son Francis may follow it up ${ }^{101}$. But I will not trouble you with any more remarks about our book.

My son will write to you about the diagram.

Let me add that I shall ever remember with pleasure your visit here last autumn ${ }^{102}$, and I remain, dear Monsieur de Candolle,

Yours very sincerely

Charles Darwin

\section{Darwin à Candolle}

My dear M. de Candolle,

July 6th [81]

I have just received a grand volume (Vol. Tertium) of the Prodromus ${ }^{103}$, which I suppose that I owe to you or your son's great kindness, and for which I am much obliged. If, however, I owe the volume to any one of the authors of the several monographs, I daresay you will have before long some communication with him and will you then be so good as to express my acknowledgements.

I have just returned from a 5 weeks holidays at our English Lakes, which are so beautiful that I think they must almost vie with the Swiss Lakes. As a consequence of my holidays I have a pile of letters to answer.

$$
\begin{aligned}
& \text { Pray believe me, } \\
& \text { Yours very sincerely, } \\
& \text { Charles Darwin }
\end{aligned}
$$

100 Movement in plants, p. 416: ... At the base of each pinna there is a pair of minute points, evidently rudiments of leaflets ... On peut supposer qu'A. DC. trouvait la conclusion hâtive. Il pourrait s'agir de glandes ou de stipules.

101 Ce travail n'a pas paru.

102 Voir ci-dessus, après la lettre du 28 août 1880.

${ }^{103}$ Il s'agit des Monographiae, et non du Prodromus. Le vol. 2, sur les Aracées, par Engler, avait paru en septembre 1879 et le $3^{\mathrm{e}}$ en juin 1881. Il contient les Philydraceae par CARuel, les Alismaceae, Butomaceae, Juncagineae par Micheli, les Commelinaceae par Clarke, et les Cucurbitacées par Cogniaux. 


\section{Candolle à Darwin}

Mon cher Monsieur,

Le 9 juillet 1881

C'est bien de notre part que vous avez reçu le volume 3 des Monographiae, quoique dans ce cas nous ayons été les éditeurs non les auteurs ${ }^{104}$. Malgré la nature purement descriptive de l'ouvrage, j'ai pensé qu'il pourrait vous servir quelquefois pour les noms les plus corrects à adopter.

Je comprends votre admiration pour les lacs d'Angleterre. Ils sont charmants - dans la bonne saison - et bien plus intéressants que ceux d'Ecosse. Hier je suis entré dans l'ancienne campagne de Sismondi, et me trouvant à l'endroit d'où Madame Darwin avait dessiné la vue, j'ai été frappé de la vérité de son dessin. Cela m'a rappelé la journée si agréable que j'ai passée à Beckenham.

Mes hommages, je vous prie à Madame Darwin, compliments à Monsieur Francis et croyez-moi toujours, mon cher Monsieur,

Votre très dévoué

Alph. de Candolle

$*$

Darwin est mort à Down le 19 avril 1882.

104 Voir ci-dessus, la liste des auteurs à la note ${ }^{103}$. 